\title{
Identification-based predictive control of semi-active shock- absorbers for adaptive dynamic excitation mitigation
}

\author{
Cezary Graczykowski (i) Rami Faraj $\mathbb{B}$
}

Received: 1 November 2019/Accepted: 4 September 2020/Published online: 20 November 2020

(C) The Author(s) 2020

\begin{abstract}
The paper is aimed at detailed discussion of the Identification-based Predictive Control (IPC) developed for semi-active fluid-based shock-absorbers which protect structures and machines against impact excitations. The problem addressed is the optimal impact absorption providing adaptive mitigation of dynamic response of the mechanical system. The goal of applied control is dissipation of the entire impact energy and minimization of the impacting object deceleration during the process. Three proposed implementations of the IPC are based on sequentially repeated procedures, which include identification of excitation parameters and calculation of the valve opening providing minimization of tracking error of the optimal path. The presented numerical examples concerning mitigation of the dynamic excitation acting on the double-chamber pneumatic shockabsorber reveal high efficiency and prove robustness of the proposed control methods. The developed algorithms are compared against each other in terms of path-tracking efficiency and character of required control actions. The most important challenges in practical implementation of the proposed methods are indicated.
\end{abstract}

C. Graczykowski $(\bowtie) \cdot$ R. Faraj

Institute of Fundamental Technological Research, Polish Academy of Sciences, Pawińskiego 5B, 02-106 Warsaw, Poland

e-mail: cezary.graczykowski@ippt.pan.pl
Keywords Adaptive control - Adaptive Impact Absorption · Identification-based Predictive Control . Model Predictive Control - Self-adaptive shockabsorber $\cdot$ Semi-active control

\section{Introduction}

Dynamic excitations are present in majority of mechanical systems and as a result the protection against shock and vibration is still an attractive field of research and development. Problems of dynamic excitation mitigation concern various systems such as aircraft landing gears [1, 2], airdrop systems [3, 4], emergency landing airbags for drones [5], suspensions of lunar-planetary landers [6], bumpers of vehicles [7, 8], road barriers [9], car airbags [10] or protection of offshore structures [11]. Large variety of shockabsorbers' applications leads to numerous technical solutions, which differ from each other in terms of construction, involved physical phenomena and complexity of control system. At present, more and more frequent practice is the use of so-called smart devices, which are based on functional materials and advanced control systems, e.g., magneto-rheological energy absorbers [12-15], electro-rheological dampers $[16,17]$, particle impact dampers [18, 19], or pneumatic adaptive absorbers with piezoelectric valves [20]. Simultaneously, advances in the development of 
control methods provide higher and higher effectiveness and reliable, adaptive performance [21-23].

The concept of Adaptive Impact Absorption (AIA) [24], which was conceived over two decades ago [25], states an important contribution to the field of dynamic excitation mitigation. Within the paradigm of AIA the control strategy applied for mitigation of the system response assumes three consecutive steps, i.e., identification of the impact excitation, calculation of optimal feasible response of the system and realization of the control scenario in order to obtain desired process of impact absorption. Such approach has been successfully applied for a number of impact absorption problems [26] and methods for identification of the impact loading can be found in literature [27]. Adaptive impact absorption is often realized with the used of semi-active absorbers, which are typically controlled in closed loop with different types of feedback, e.g., force [28] or acceleration feedback [29]. Following the AIA approach the value of the absorber reaction force or alternatively the piston deceleration is calculated based on the identified values of excitation parameters [30]. Then, the system tracks the calculated path until stopping of the impacting object.

Design of control systems for the AIA approach can be based on classical methods such as well-known PID controllers [31-33] or their modern modifications such as self-regulating fuzzy PD controllers [34]. The disadvantage of the classical approach is the fact that system relies on the quality of initial excitation identification and robustness to possible disturbances in the impact absorption process is not provided. This problem was addressed by the authors in previous papers, where consequences of imprecise initial identification of the excitation has been revealed [35], the method providing self-adaptive performance of the fluid-based absorbers has been introduced [36] and experimentally validated using drop tests [37].

This paper proposes new control methods, which are based on the identification of excitation parameters, but in contrast to the original AIA approach the identification is not treated as a separate process performed before single calculation of the control strategy at the beginning of impact absorption process. On the contrary, system performs iterative identification of excitation parameters and updates the control signal at every control step.
For the sake of clarity the proposed methods are discussed on the illustrative example of doublechamber pneumatic shock-absorbers, which together with hydraulic dampers, represent the wider group of semi-active fluid-based absorbers and constitute one of the most basic devices used for adaptive impact absorption. Such absorbers are typically composed of two sealed chambers filled with pneumatic or hydraulic fluid and the controllable (e.g. electromechanical or piezo-electric) valve. In addition, they are equipped with system of sensors, which enables measurement of the actual state of the fluid (pressure and temperature of gas in both chambers) and actual kinematics of the piston (its acceleration or displacement with respect to the device compartment). Moreover, semi-active shock-absorbers are equipped with fast controllers, which enable real-time modification of the actual valve opening and control of the actual rate of the fluid flow between the chambers. Semiactive shock-absorbers are typically subjected to two different types of dynamic excitations: i) impact excitation caused by object hitting the piston with initial velocity, ii) external force applied to the piston. General scheme of semi-active fluid-based shockabsorber considered in further part of this study is presented in Fig. 1.

In the case of impact excitation, the objective of semi-active shock-absorber is to dissipate the entire impact energy and mitigate the dynamic system response by minimization of the force generated by the absorber and minimization of the impacting object deceleration. In general, such objective can be achieved by the adjustment of applied level of generated absorber force to determined impact energy and by using real-time control of the valve opening to provide the required mass flow rate of fluid between both chambers of the device. In the case of hydraulic shock-absorber, the optimal strategy of impact absorption provides dissipation of the entire impact energy with constant value of force maintained until the end of the stroke. In turn, in the case of pneumatic shockabsorber, the strategy of optimal impact absorption requires initial stage of force increase and the stage when force remains constant until the end of the process. As a result of applied paradigm of adaptation and real-time control, the semi-active fluid-based hydraulic and pneumatic shock-absorbers can be adjusted to actual dynamic excitation and they surpass 
Fig. 1 General scheme of semi-active fluid-based shock-absorber [38]

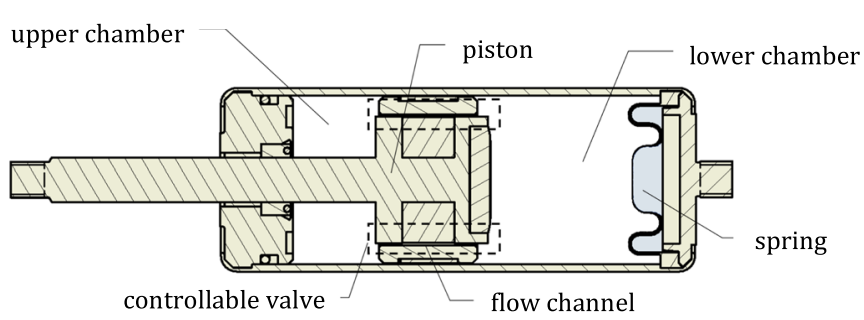

classical passive shock-absorbers in terms of efficiency and operation robustness.

\section{Problem of dynamic excitation mitigation}

\subsection{Exact and simplified mathematical model of the system}

The basis for development of identification-based semi-active control methods is exact mathematical model of the fluid-based shock-absorber, which is based on the so-called concept of physical modelling [38]. In this approach the response of the shockabsorber is described exclusively with the use of fundamental physical principles, while no phenomenological relations describing global response and value of generated resistance force are used. In particular, in the considered case of the doublechamber fluid-based shock-absorber (Fig. 2) the most fundamental one degree-of-freedom model of the analysed impact absorbing system is based on equation of motion of the impacting object, mass balance equations for the fluid enclosed in both chambers combined with equation of the valve flow, thermodynamic energy balance equations for the fluid in both chambers and equations of state of the fluid.

For the sake of simplicity we consider typical loading scenario when the right chamber of the device is compressed and the left chamber is decompressed. As a result the gas flows from the right (upstream) chamber to left (downstream) chamber. The fundamental version of the system of equations governing the problem of impact absorption takes the form:

$$
\begin{aligned}
& \mathrm{Mü}+\left(\mathrm{p}_{2} \mathrm{~A}_{2}-\mathrm{p}_{1} \mathrm{~A}_{1}\right)+\mathrm{F}_{\text {dist }}=\mathrm{F}_{\mathrm{ext}} \\
& \dot{\mathrm{m}}_{1}=\mathrm{Q}_{\mathrm{m}}=\mathrm{A}_{v}(\mathrm{t}) \sqrt{\frac{2 \kappa}{\kappa-1}\left(\mathrm{q}^{\frac{2}{\kappa}}-\mathrm{q}^{\frac{\kappa+1}{\kappa}}\right)} \frac{\mathrm{p}_{2}}{\sqrt{\mathrm{RT}_{2}}} \\
& \dot{\mathrm{m}}_{2}=-\mathrm{Q}_{\mathrm{m}} \\
& \dot{\mathrm{m}}_{1} \mathrm{c}_{\mathrm{p}} \mathrm{T}_{2}+\dot{\mathrm{Q}}_{1}=\dot{\mathrm{m}}_{1} \mathrm{c}_{\mathrm{v}} \mathrm{T}_{1}+\mathrm{m}_{1} \mathrm{c}_{\mathrm{v}} \dot{\mathrm{T}}_{1}+\mathrm{p}_{1} \dot{\mathrm{V}}_{1} \\
& \dot{\mathrm{m}}_{2} \mathrm{c}_{\mathrm{p}} \mathrm{T}_{2}+\dot{\mathrm{Q}}_{2}=\dot{\mathrm{m}}_{2} \mathrm{c}_{\mathrm{v}} \mathrm{T}_{2}+\mathrm{m}_{2} \mathrm{c}_{\mathrm{v}} \dot{\mathrm{T}}_{2}+\mathrm{p}_{2} \dot{\mathrm{V}}_{2} \\
& \mathrm{p}_{1} \mathrm{~V}_{1}=\mathrm{m}_{1} \mathrm{RT}_{1}, \mathrm{p}_{2} \mathrm{~V}_{2}=\mathrm{m}_{2} \mathrm{RT}_{2} \\
& \mathrm{~V}_{1}=\mathrm{A}_{1}\left(\mathrm{~h}_{1}^{0}+\mathrm{u}\right), \mathrm{V}_{2}=\mathrm{A}_{2}\left(\mathrm{~h}_{2}^{0}-\mathrm{u}\right) \\
& \mathrm{IC}: \mathrm{u}(0)=\mathrm{u}_{0}, \dot{\mathrm{u}}(0)=\mathrm{v}_{0}, \mathrm{p}_{1}(0)=\mathrm{p}_{1}^{0}, \\
& \mathrm{p}_{2}(0)=\mathrm{p}_{2}^{0}, \mathrm{~T}_{1}(0)=\mathrm{T}_{1}^{0}, \mathrm{~T}_{2}(0)=\mathrm{T}_{2}^{0}
\end{aligned}
$$

where $\mathrm{M}$ is the total mass of the impacting object and the piston, $\mathrm{u}$ is displacement of the piston with respect to the compartment of the device, $\mathrm{F}_{\text {dist }}$ is the disturbance force (typically not a priori known and corresponding to a sum of various unconsidered forces, e.g. friction of unknown value) and $F_{e x t}$ is a timedependent external force acting on the impacting object or the piston. The parameters of gas include pressures $\left(\mathrm{p}_{1}\right.$ and $\left.\mathrm{p}_{2}\right)$, temperatures $\left(\mathrm{T}_{1}\right.$ and $\left.\mathrm{T}_{2}\right)$ and mass of gas $\left(\mathrm{m}_{1}\right.$ and $\left.\mathrm{m}_{2}\right)$ in decompressed and
Fig. 2 Considered doublechamber pneumatic absorber subjected to impact excitation

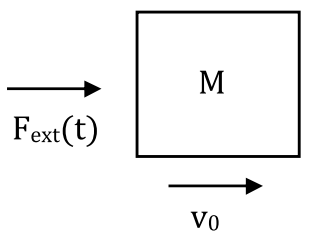

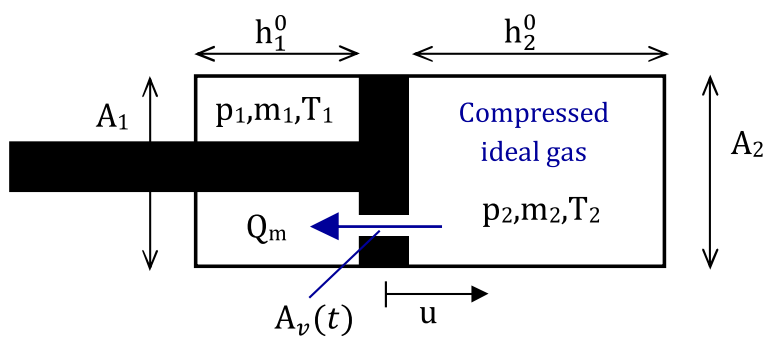


compressed chamber of the shock-absorber. The quantity $\mathrm{Q}_{\mathrm{m}}$ denotes mass flow rate of gas from upstream to downstream chamber, approximated by isentropic flow model, which depends on the actual area of the valve opening $\mathrm{A}_{v}(\mathrm{t})$ and the quantity $\mathrm{q}$ equal to $\frac{\mathrm{p}_{1}}{\mathrm{p}_{2}}$ for the subsonic flow or $\left(\frac{2}{\kappa+1}\right)^{\frac{\kappa}{\kappa-1}}$ for the choked flow. The equations of energy balance (Eqs. 4, 5 ) indicate that sum of enthalpy of gas transferred to each chamber and inflow of heat $\left(\dot{\mathrm{Q}}_{1}\right.$ and $\left.\dot{\mathrm{Q}}_{2}\right)$ equals to increase of gas internal energy and work done by gas. The quantity $\mathrm{R}$ is a gas constant, while $\kappa$ denotes adiabatic exponent defined as ratio of specific heat at constant pressure $c_{p}$ and specific heat at constant volume $c_{\mathrm{V}}$. The actual volumes of decompressed and compressed chamber $\left(\mathrm{V}_{1}\right.$ and $\left.\mathrm{V}_{2}\right)$ are expressed in terms of piston displacement $\mathrm{u}$, chambers crosssectional areas $A_{1}$ and $A_{2}$ and their initial lengths $h_{1}^{0}$ and $h_{2}^{0}$. According to the above form of the governing equations (five differential and four algebraic ones) the system has six state variables, which can be selected for example as: displacement and velocity of the piston, masses and temperatures of gas in each chamber of the device. The control variable of the problem is actual area of valve opening connecting the absorber's chambers $\mathrm{A}_{v}(\mathrm{t})$.

The significant simplification of the mathematical model can be obtained by summation and integration of the selected governing differential equations in order to obtain their algebraic counterparts. In the case of differential equations governing balance of fluid mass in each chamber, the algebraic equation defining conservation of fluid mass in the whole absorber is obtained directly. In turn, in the case of differential equations of motion and thermodynamic energy balance, the algebraic equation defining global energy balance is obtained exclusively for the system subjected to impact defined by the initial conditions $\left(\mathrm{F}_{\mathrm{ext}}=\right.$ $0, F_{\text {dist }}=0$ ) or system with constant value of external and disturbance force $\left(\mathrm{F}_{\mathrm{ext}}=\right.$ const., $\mathrm{F}_{\mathrm{dist}}=$ const. $)$, which operates in adiabatic conditions $\left(\dot{\mathrm{Q}}_{1}=\dot{\mathrm{Q}}_{2}=0\right)$. In such a case, the simplified system of equations takes the form:

$\mathrm{Mü}+\left(\mathrm{p}_{2} \mathrm{~A}_{2}-\mathrm{p}_{1} \mathrm{~A}_{1}\right)+\mathrm{F}_{\mathrm{dist}}=\mathrm{F}_{\mathrm{ext}}$

$$
\begin{aligned}
& \dot{\mathrm{m}}_{1}=\mathrm{A}_{v}(\mathrm{t}) \sqrt{\frac{2 \kappa}{\kappa-1}\left(\mathrm{q}^{\frac{2}{\kappa}}-\mathrm{q}^{\frac{\kappa+1}{\kappa}}\right)} \frac{\mathrm{p}_{2}}{\sqrt{\mathrm{RT}_{2}}} \\
& \mathrm{~m}_{1}+\mathrm{m}_{2}=\mathrm{m}
\end{aligned}
$$

$$
\begin{aligned}
& \frac{1}{2} \mathrm{Mv}_{0}^{2}-\frac{1}{2} \mathrm{Mv}^{2}+\left(\mathrm{F}_{\mathrm{ext}}-\mathrm{F}_{\text {dist }}\right)\left(\mathrm{u}-\mathrm{u}_{0}\right) \\
& \quad=\mathrm{m}_{1} \mathrm{c}_{\mathrm{v}} \mathrm{T}_{1}+\mathrm{m}_{2} \mathrm{c}_{\mathrm{v}} \mathrm{T}_{2}-\mathrm{m}_{1}^{0} \mathrm{c}_{\mathrm{v}} \mathrm{T}_{1}^{0}-\mathrm{m}_{2}^{0} \mathrm{c}_{\mathrm{v}} \mathrm{T}_{2}^{0}
\end{aligned}
$$

$\frac{\mathrm{p}_{2} \mathrm{~V}_{2}^{\kappa}}{\mathrm{m}_{2}^{\kappa}}=\frac{\mathrm{p}_{2}^{0}\left(\mathrm{~V}_{2}^{0}\right)^{\kappa}}{\left(\mathrm{m}_{2}^{0}\right)^{\kappa}}=$ const.

$\mathrm{p}_{1} \mathrm{~V}_{1}=\mathrm{m}_{1} \mathrm{RT}_{1}, \mathrm{p}_{2} \mathrm{~V}_{2}=\mathrm{m}_{2} \mathrm{RT}_{2}$

$\mathrm{V}_{1}=\mathrm{A}_{1}\left(\mathrm{~h}_{1}^{0}+\mathrm{u}\right), \mathrm{V}_{2}=\mathrm{A}_{2}\left(\mathrm{~h}_{2}^{0}-\mathrm{u}\right)$

IC $: \mathrm{u}(0)=\mathrm{u}_{0}, \dot{\mathrm{u}}(0)=\mathrm{v}_{0}, \mathrm{~m}_{1}(0)=\mathrm{m}_{1}^{0}$

Let us note that above version of the mathematical model is composed of two differential equations governing motion of the piston and mass transfer of gas between the chambers, and seven algebraic equations defining additional dependencies between gas parameters and definitions of chambers volumes. Consequently, the system is described by three state variables: displacement and velocity of the piston and mass of gas in decompressed chamber. The initial conditions are imposed exclusively on these three variables.

In the proposed solution procedure the algebraic equations are solved analytically and, as a result, all state parameters of gas are expressed as functions of displacement and velocity of the piston, and mass of the gas in decompressed chamber. Further, the resulting system of two nonlinear differential equations is solved numerically using forward Euler method or 4-5th order Runge-Kutta method. The above simplified system of governing equations will be particularly useful in development of Identification-based Predictive Control strategies presented in the following sections.

\subsection{Control problem formulation and its classical solution}

As previously mentioned, adaptation of semi-active fluid-based absorber is based on real-time modification 
of the valve opening and it is aimed at absorption and dissipation of the entire impact energy with minimal level of force generated on the impacting object and minimal resulting value of its deceleration. Therefore, the standard straightforward formulation of the optimal impact absorption problem reads:

Find $\mathrm{A}_{v}(\mathrm{t}) \mid \int_{\mathrm{u}_{0}}^{\mathrm{u}(\mathrm{T})} \mathrm{F}_{\mathrm{abs}} \mathrm{du}=\mathrm{E}_{\mathrm{imp}}$

and $\max \left(\mathrm{F}_{\mathrm{abs}}-\mathrm{F}_{\mathrm{ext}}\right)$ is minimal

subject to: model describing system dynamics defined by Eqs. $1-10$.

The variable $\mathrm{T}$ is the final time of the process when the state of static equilibrium of the impacting object is achieved $\left(\mathrm{F}_{\mathrm{ext}}=\mathrm{F}_{\mathrm{dist}}=0, \mathrm{~F}_{\mathrm{abs}}=0\right)$, while $\mathrm{F}_{\mathrm{abs}}$ is the total force generated by the absorber defined as a sum of pneumatic and disturbance force: $F_{a b s}=F_{\text {pneu }}+$ $F_{\text {dist }}$. Moreover, $E_{i m p}$ is the total energy to be dissipated, being a sum of initial energy of the impacting object and work done by the external force:

$\mathrm{E}_{\mathrm{imp}}=\mathrm{E}_{\mathrm{imp}}^{0}+\mathrm{E}_{\mathrm{imp}}^{\mathrm{ext}}=\frac{1}{2} \mathrm{Mv}_{0}^{2}+\int_{\mathrm{u}_{0}}^{\mathrm{u}(\mathrm{T})} \mathrm{F}_{\text {ext }} \mathrm{du}$

where $\mathrm{u}(\mathrm{T}) \leq \mathrm{d}$ and $\mathrm{d}$ is an assumed absorber's stroke $\mathrm{d} \leq \mathrm{h}_{2}^{0}$. The alternative formulation of the optimal impact absorption problem has the form of pathtracking problem:

Find $\mathrm{A}_{v}(\mathrm{t}) \mid \int_{\mathrm{u}_{0}}^{\mathrm{u}(\mathrm{T})} \mathrm{F}_{\mathrm{abs}} \mathrm{du}=\mathrm{E}_{\mathrm{imp}}$

and $\int_{\mathrm{t}_{0}}^{\mathrm{T}}\left(\mathrm{F}_{\mathrm{abs}}(\mathrm{t})-\mathrm{F}_{\mathrm{abs}}^{\mathrm{opt}}\right)^{2} \mathrm{dt}$ is minimal

where $F_{a b s}^{o p t}$ is an optimal constant value of force generated by the absorber and it results from total amount of submitted energy $E_{\text {imp }}$ and assumed absorber's stroke d:

$\mathrm{F}_{\mathrm{abs}}^{\mathrm{opt}}=\frac{\mathrm{Mv}_{0}^{2}+2 \int_{\mathrm{u}_{0}}^{\mathrm{d}} \mathrm{F}_{\mathrm{ext}} \mathrm{du}}{2 \mathrm{~d}}$

The standard solution of impact mitigation problem is based on three strict simplifying assumptions: i) impacting object mass, its velocity and applied external force are either known or identified at the beginning of the process, ii) no disturbances (such as additional unknown forces or fluid leakages) are present in the system, iii) no constraints on maximal valve opening or maximal speed of valve operation are considered (the relevant values and changes of the gas mass flow rate through the valve are provided). Under such assumptions the optimal control strategy is typically obtained in two steps. The first step is solution of the path-finding problem aimed at finding optimal feasible (realizable) change of the absorber force $\mathrm{F}_{\mathrm{abs}}^{\text {feas }}(\mathrm{u})$ :

Find $F_{\text {abs }}^{\text {feas }}(u) \mid \int_{u_{0}}^{u(T)} F_{\text {abs }}^{\text {feas }} \mathrm{du}=E_{\text {imp }}$

and $\int_{\mathrm{u}_{0}}^{\mathrm{u}(\mathrm{T})}\left(\mathrm{F}_{\mathrm{abs}}^{\mathrm{feas}}(\mathrm{u})-\mathrm{F}_{\mathrm{abs}}^{\mathrm{opt}}\right)^{2} \mathrm{du}$ is minimal

subject to: model describing system dynamics defined by Eqs. 1-10.

The force $\mathrm{F}_{\mathrm{abs}}^{\mathrm{feas}}(\mathrm{u})$ is computed taking into account that fluid is compressible and initial increase of force cannot be immediate, but it requires utilization of at least the part of absorber stroke. For the case of double-chamber pneumatic shock-absorber the solution of the above path-finding problem includes three distinct stages: (i) possibly fast increase of pneumatic force with a closed valve, (ii) maintaining constant value of pneumatic force, (iii) reduction of generated force to zero to provide static equilibrium. Taking into account the adiabatic equation of state for the gas of constant mass (simplified version of Eq. 15) the above three stages can be defined as:

$\mathrm{F}_{\mathrm{abs}}^{\mathrm{feas}}(\mathrm{u})=\mathrm{A}_{2} \mathrm{p}_{2}^{0}\left(\frac{\mathrm{V}_{2}^{0}}{\mathrm{~V}_{2}(\mathrm{u})}\right)^{\kappa}-\mathrm{A}_{1} \mathrm{p}_{1}^{0}\left(\frac{\mathrm{V}_{1}^{0}}{\mathrm{~V}_{1}(\mathrm{u})}\right)^{\kappa}$,

$\mathrm{F}_{\text {abs }}^{\text {feas }}(\mathrm{u})=$ const., $\quad \mathrm{F}_{\text {abs }}^{\text {feas }}(\mathrm{u}) \rightarrow 0$

The second stage is based on the solution of the pathtracking problem aimed at determination of variable valve opening, which allows to follow previously determined feasible force path:

Find $\mathrm{A}_{v} \mid \int_{0}^{\mathrm{T}}\left(\mathrm{F}_{\mathrm{abs}}\left(\mathrm{A}_{v}\right)-\mathrm{F}_{\mathrm{abs}}^{\mathrm{feas}}\right)^{2} \mathrm{dt}$ is minimal 
The above problem is often solved using the so-called inverse dynamics approach, which assumes ideal tracking of the feasible path and enables computation of valve opening using the system of governing equations (Eqs. 11-20) supplemented with the additional condition $\mathrm{F}_{\mathrm{abs}}\left(\mathrm{A}_{v}\right)=\mathrm{F}_{\mathrm{abs}}^{\text {feas }}$. The solution of the path-tracking problem can be defined: i) in terms of displacement of the piston $\mathrm{A}_{v}(\mathrm{u})$ - semi-passive or semi-active control system [39], ii) directly in terms of time $\mathrm{A}_{v}(\mathrm{t})$-open-loop control system, iii) in terms of piston velocity $\mathrm{A}_{v}(\mathrm{v})$-feedback control system. Eventually, the third stage assumes reduction of absorber's force to zero in order to obtain the state of static equilibrium of the system and dissipation of the entire impact energy. In the case when almost entire admissible stroke is utilized and the valve efficiency is large the third stage can be executed infinitely fast. It includes immediate equalization of pressures in both chambers and infinitesimal backward motion of the piston. Three above stages obtained within the classical AIA approach are visualized in Fig. 3a and b, respectively.

The evident drawback of the classical AIA approach is the fact that it requires either prior knowledge of actual excitation, which is very rare in most practical situations or its immediate identification at the beginning of the process. Moreover, the algorithm is characterized by the lack of robustness to subsequent impact and system disturbances. Finally, it will not operate properly in the case of active constraints imposed on maximal valve opening or its maximal operation speed. Most of these drawbacks are eliminated by the proposed Identification-based Predictive Control algorithms, which will ensure adaptive

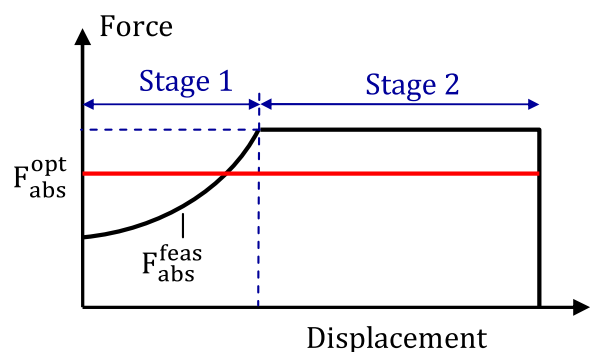

(a) and robust performance of semi-active fluid-based absorbers.

\section{The concept and theory of self-adaptive systems}

This section discusses the concept of self-adaptive systems and derivation of the variational formulations being the basis for development of the Identificationbased Predictive Control (IPC) systems. We focus on the problem of impact mitigation, in which dynamic excitation applied to the shock-absorber is defined by impacting object mass and its initial velocity ( $\mathrm{M}$ and $v_{0}$ ) or external force $F_{\text {ext }}(t)$ in the form of impulses of relatively short duration.

The Identification-based Predictive Control algorithms implemented in the absorbers allow for obtaining high and robust performance and classify them within the group of self-adaptive impact absorbing systems, which do not require preliminary knowledge about dynamic excitation and despite this fact they are able to automatically adapt to actual conditions and provide optimal dissipation of the impact energy. Consequently, the absorbers equipped with the IPC should meet strict requirements formulated for self-adaptive systems and provide:

1. Automatic adaptation to unknown excitations even if they repeat during the process.

2. Robustness to unknown disturbances, e.g., additional forces or sudden leakages of the fluid.

The above demands significantly complicate elaboration of relevant control strategy and cause that application of the above described two stage procedure, based on subsequent steps of path-finding and path-tracking, cannot be directly applied.

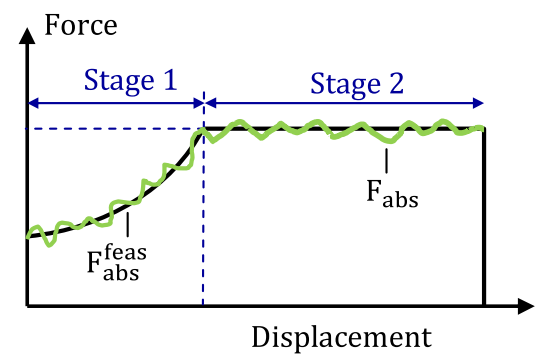

(b)

Fig. 3 Standard procedure applied for finding the optimal valve opening of the double-chamber shock-absorber: a the stage of pathfinding, $\mathbf{b}$ the stage of path-tracking 
3.1 Mathematical formulations of optimal selfadaptive impact mitigation problems

Mathematical formulation of the impact mitigation problem referring to self-adaptive system is substantially different than in the classical approach. The derivation of the variational formulation of the control problem for self-adaptive absorbers is based on general principle of their operation, which includes on-line adaptation by determination of the currently optimal system path. The fundamental idea is that initial conditions and entire time-history of excitation, disturbances and control applied so far are reflected in the actual system kinematics. Consequently, the knowledge of actual system kinematics can be used for determination of the optimal system path for the further part of the process. Such approach allows to determine actual optimal value of reaction force $F_{a b s}^{\text {opt }}$ and corresponding value of acceleration $\mathrm{a}^{\mathrm{opt}}$, which should be maintained constant until the end of the stroke in order to provide absorption of the entire impact energy.

In particular, the force $\mathrm{F}_{\mathrm{abs}}^{\mathrm{opt}}$ can be computed by integration of the equation of motion (Eq. 11) over displacement from actual piston position $u(t)$ to the end of available stroke $d$, under assumption that force generated by the absorber and external force remain constant until the end of the process:

$$
\int_{u(t)}^{d} M u ̈ d u+\int_{u(t)}^{d} F_{a b s}^{o p t} d u=\int_{u(t)}^{d} F_{e x t} d u
$$

As a result, we obtain the actual optimal value of absorber's reaction force:

$\mathrm{F}_{\mathrm{abs}}^{\mathrm{opt}}(\mathrm{t})=\frac{\mathrm{Mü}(\mathrm{t})^{2}}{2(\mathrm{~d}-\mathrm{u}(\mathrm{t}))}+\mathrm{F}_{\mathrm{ext}}(\mathrm{t})$

Using Eq. 11 once again, we obtain purely kinematic condition, further called Kinematic Optimality Condition (KOC), which defines actual optimal value of acceleration:

$\mathrm{a}^{\mathrm{opt}}(\mathrm{t})=-\frac{\dot{\mathrm{u}}(\mathrm{t})^{2}}{2(\mathrm{~d}-\mathrm{u}(\mathrm{t}))}$
The above conditions enable introducing two variational formulations of state-dependent path-tracking problem: (i) the force-based formulation and (ii) the kinematics-based formulation.

\subsection{Force-based state-dependent path-tracking}

General form of force-based state-dependent pathtracking problem assumes minimization of the squared difference between total force generated by the absorber $\mathrm{F}_{\mathrm{abs}}\left(\mathrm{A}_{v}(\mathrm{t}), \mathrm{t}\right)$ and the actual optimal value of force $F_{a b s}^{o p t}(t)$ :

Find $\mathrm{A}_{v}(\mathrm{t}) \mid \int_{\mathrm{u}_{0}}^{\mathrm{u}(\mathrm{T})} \mathrm{F}_{\mathrm{abs}} \mathrm{du}=\mathrm{E}_{\text {imp }}$
and $\int_{0}^{\mathrm{T}}\left(\mathrm{F}_{\mathrm{abs}}\left(\mathrm{A}_{v}(\mathrm{t}), \mathrm{t}\right)-\mathrm{F}_{\mathrm{abs}}^{\mathrm{opt}}(\mathrm{t})\right)^{2} \mathrm{dt}$ is minimal

subject to: model describing system dynamics defined by Eqs. 11-20.

The total absorber force generated by the absorber is defined as:

$\mathrm{F}_{\text {abs }}\left(\mathrm{A}_{v}(\mathrm{t}), \mathrm{t}\right)=\mathrm{F}_{\text {pneu }}\left(\mathrm{A}_{v}(\mathrm{t})\right)+\mathrm{F}_{\text {dist }}(\mathrm{t})$

while the actual optimal absorber force $\mathrm{F}_{\mathrm{abs}}^{\mathrm{opt}}$ is given by Eq. 29. The above formulation substantially differs from the classical path-tracking problem (Eq. 27) as the second term depends on actual state of the system. Since the absorber force $\mathrm{F}_{\mathrm{abs}}$ depends on disturbance force $F_{\text {dist }}$, while the optimal absorber force $F_{a b s}^{\text {opt }}$ depends on external force $\mathrm{F}_{\text {ext }}$ and both these forces are assumed to be unknown, the direct solution of the state-dependent path-tracking problem cannot be found. However, by applying time-discretization the above problem can be transformed into series of pathtracking problems defined for time intervals $\left[\mathrm{t}_{\mathrm{i}}, \mathrm{T}\right]$, while application of the model predictive control/ receding horizon control (MPC/RHC) enables shortening the control interval. Moreover, the actual difference of unknown forces $F_{\text {ext }}$ and $F_{\text {dist }}$ can be calculated using impacting object's equation of motion at initial time instant of each control step. Such approach leads to force-kinematics state- 
dependent path-tracking problem, which is the basis for the Hybrid Prediction Control method developed in previous paper of the authors [36].

The additional comment concerns absorption and dissipation of the impact energy. The optimal force $\mathrm{F}_{\mathrm{abs}}^{\mathrm{opt}}(\mathrm{t})$ corresponds to absorption of the entire impact energy so the condition of energy absorption is fulfilled by reaching actual optimal path just before the end of the process. Since in the considered unconstrained problem the final part of the optimal path can be always precisely tracked, the integral condition of energy absorption will be always met and it can be removed from problem formulation. In turn, the condition of energy dissipation requires obtaining the state of static equilibrium at assumed stroke d, when impacting object is stopped. For the considered purely pneumatic absorber with unequal areas of both chambers such state can be obtained only when $\mathrm{d} \rightarrow \mathrm{h}_{2}^{0}$. In case $\mathrm{d}<\mathrm{h}_{2}^{0}$ the problem formulation defined by Eq. 31 has to be weakened and the condition of energy dissipation has to be replaced by the condition of energy absorption in which $\mathrm{T}$ denotes time instant when $\mathrm{v}=0$.

\subsection{Kinematics-based state-dependent path- tracking}

The problem of optimal impact absorption can be also expressed in terms of kinematic quantities. The kinematics-based state-dependent path-tracking problem can be formulated directly by assuming minimization of the squared difference between predicted and actual optimal value of impacting object acceleration, i.e.:

Find $\mathrm{A}_{v}(\mathrm{t}) \mid \int_{0}^{\mathrm{T}}\left(\ddot{\mathrm{u}}\left(\mathrm{A}_{v}(\mathrm{t}), \mathrm{t}\right)-\mathrm{a}^{\mathrm{opt}}(\mathrm{t})\right)^{2} \mathrm{dt}$ is minimal

Alternatively, it can be derived from the force-based state-dependent path-tracking problem (Eq. 31) using definition of total absorber force (Eq. 32), definition of the actual optimal force (Eq. 29) and introducing the difference of external and disturbance forces obtained from equation of motion (Eq. 11):

$$
\mathrm{F}_{\text {ext }}(\mathrm{t})-\mathrm{F}_{\text {dist }}(\mathrm{t})=\mathrm{Mü}(\mathrm{t})+\mathrm{F}_{\text {pneu }}\left(\mathrm{A}_{v}(\mathrm{t})\right)
$$

This leads to the formulation:
Find $\mathrm{A}_{v}(\mathrm{t}) \mid \int_{0}^{\mathrm{T}}\left(\ddot{\mathrm{u}}\left(\mathrm{A}_{v}(\mathrm{t}), \mathrm{t}\right)+\frac{\dot{\mathrm{u}}(\mathrm{t})^{2}}{2(\mathrm{~d}-\mathrm{u}(\mathrm{t}))}\right)^{2} \mathrm{dt}$

is minimal

The formulations given by (Eq. 33) and (Eq. 35) are obviously equivalent, which results from definition of the actual optimal acceleration value (Eq. 30). Similarly as in the case of force-based formulation the proposed solution method is based on discretization of the state-dependent path-tracking problem and sequential solution of the series of the problems formulated for time intervals $\left[\mathrm{t}_{\mathrm{i}}, \mathrm{T}\right]$ :

Find $\mathrm{A}_{v}(\mathrm{t}) \mid \int_{\mathrm{t}_{\mathrm{i}}}^{\mathrm{T}}\left(\ddot{\mathrm{u}}\left(\mathrm{A}_{v}(\mathrm{t}), \mathrm{t}\right)+\frac{\dot{\mathrm{u}}\left(\mathrm{t}_{\mathrm{i}}\right)^{2}}{2\left(\mathrm{~d}-\mathrm{u}\left(\mathrm{t}_{\mathrm{i}}\right)\right)}\right)^{2} \mathrm{dt}$ isminimal

The computational cost is reduced by shortening the applied control interval into the length of a single control step $\left[\mathrm{t}_{\mathrm{i}}, \mathrm{t}_{\mathrm{i}}+\Delta \mathrm{t}\right]$. As a result, each subsequent control problem takes the form:

Find $\mathrm{A}_{v}(\mathrm{t}) \mid \int_{\mathrm{t}_{\mathrm{i}}}^{\mathrm{t}_{\mathrm{i}}+\Delta \mathrm{t}}\left(\ddot{\mathrm{u}}\left(\mathrm{A}_{v}(\mathrm{t}), \mathrm{t}\right)+\frac{\dot{\mathrm{u}}\left(\mathrm{t}_{\mathrm{i}}\right)^{2}}{2\left(\mathrm{~d}-\mathrm{u}\left(\mathrm{t}_{\mathrm{i}}\right)\right)}\right)^{2} \mathrm{dt}$

is minimal

Let us note that in the above formulation the integral corresponds to a single control step, but the definition of the value of optimal deceleration refers to the optimal path for the entire remaining part of the impact absorption process. Thus, the above variational formulation can be considered as local-global approach. The above formulation is a classical path-tracking problem, in which the second term is constant. In contrast to force-based formulation it does not contain the external and disturbance forces so it does not require further modifications and reformulations. Nevertheless, the values of external and disturbance forces are required for computation of the actual value of impacting object deceleration. Consequently, identification of the external and disturbance forces at beginning of each control step will be required. They will be used to derive a predictive model applied for simulation of system response and determination of the optimal valve opening. 


\subsection{Identification-based predictive control}

In the further part of the paper three different implementations of the Identification-based Predictive Control (IPC) are discussed. Their development is focused on more precise control of valve opening and more accurate tracking of the optimal system path than achieved by previously developed methods such as the Hybrid Prediction Control [36]. In particular, they should ensure correct operation of the shock-absorber when initial deceleration is close to the optimal one.

This objective is achieved by identification of the difference of external and disturbance force at the beginning of each control step by recalling the equation of impacting object motion (Eq. 11) at time instant $t_{i}$, which yields:

$\mathrm{F}_{\text {ext }}\left(\mathrm{t}_{\mathrm{i}}\right)-\mathrm{F}_{\text {dist }}\left(\mathrm{t}_{\mathrm{i}}\right)=\mathrm{Mü}\left(\mathrm{t}_{\mathrm{i}}\right)+\mathrm{F}_{\text {pneu }}\left(\mathrm{t}_{\mathrm{i}}\right)$

Identified force difference is assumed to remain constant during the entire control step and to change at the subsequent control steps of the process. Conducted identification enables development of the predictive model, which is implemented in the controller in order to simulate and optimize system response at a single control step. Such model is obtained from simplified model of the system defined by Eqs. 11-20 by assuming $\mathrm{F}_{\text {ext }}(\mathrm{t})-\mathrm{F}_{\text {dist }}(\mathrm{t})=$ $\mathrm{F}_{\text {ext }}\left(\mathrm{t}_{\mathrm{i}}\right)-\mathrm{F}_{\text {dist }}\left(\mathrm{t}_{\mathrm{i}}\right)$ and by substituting Eq. 38. This leads to specific forms of the equation of motion (Eq. 39) and equation of global energy balance (Eq. 42). The system of equations for a single control step takes the form:

$$
\begin{aligned}
& \mathrm{M}\left(\ddot{\mathrm{u}}-\ddot{\mathrm{u}}\left(\mathrm{t}_{\mathrm{i}}\right)\right)+\left(\mathrm{p}_{2}-\mathrm{p}_{2}\left(\mathrm{t}_{\mathrm{i}}\right)\right) \mathrm{A}_{2}-\left(\mathrm{p}_{1}-\mathrm{p}_{1}\left(\mathrm{t}_{\mathrm{i}}\right)\right) \mathrm{A}_{1}=0 \\
& \dot{\mathrm{m}}_{1}=\mathrm{A}_{v}(\mathrm{t}) \sqrt{\frac{2 \kappa}{\kappa-1}\left(\mathrm{q}^{\frac{2}{\kappa}}-\mathrm{q}^{\frac{\kappa+1}{\kappa}}\right)} \frac{\mathrm{p}_{2}}{\sqrt{\mathrm{RT}_{2}}} \\
& \mathrm{~m}_{1}+\mathrm{m}_{2}=\mathrm{m} \\
& \frac{1}{2} \mathrm{Mv}\left(\mathrm{t}_{\mathrm{i}}\right)^{2}-\frac{1}{2} \mathrm{Mv}^{2} \\
& \left.+\left[\mathrm{Mu}_{\mathrm{i}}\right)+\left(\mathrm{p}_{2}\left(\mathrm{t}_{\mathrm{i}}\right) \mathrm{A}_{2}-\mathrm{p}_{1}\left(\mathrm{t}_{\mathrm{i}}\right) \mathrm{A}_{1}\right)\right]\left(\mathrm{u}-\mathrm{u}\left(\mathrm{t}_{\mathrm{i}}\right)\right) \\
& =\frac{\mathrm{p}_{1} \mathrm{~V}_{1}}{\kappa-1}+\frac{\mathrm{p}_{2} \mathrm{~V}_{2}}{\kappa-1}-\frac{\mathrm{p}_{1}\left(\mathrm{t}_{\mathrm{i}}\right) \mathrm{V}_{1}\left(\mathrm{t}_{\mathrm{i}}\right)}{\kappa-1}-\frac{\mathrm{p}_{2}\left(\mathrm{t}_{\mathrm{i}}\right) \mathrm{V}_{2}\left(\mathrm{t}_{\mathrm{i}}\right)}{\kappa-1}
\end{aligned}
$$

$\frac{\mathrm{p}_{2} \mathrm{~V}_{2}^{\kappa}}{\mathrm{m}_{2}^{\kappa}}=\frac{\mathrm{p}_{2}\left(\mathrm{t}_{\mathrm{i}}\right) \mathrm{V}_{2}\left(\mathrm{t}_{\mathrm{i}}\right)^{\kappa}}{\mathrm{m}_{2}\left(\mathrm{t}_{\mathrm{i}}\right)^{\kappa}}$

IC $: \mathrm{u}\left(\mathrm{t}_{\mathrm{i}}\right)=\mathrm{u}_{0}, \dot{\mathrm{u}}\left(\mathrm{t}_{\mathrm{i}}\right)=\mathrm{v}_{0}, \mathrm{~m}_{1}\left(\mathrm{t}_{\mathrm{i}}\right)=\mathrm{m}_{1}^{0}$

and it is complemented by equation of state of gas in both chambers (Eqs. 16, 17) and definitions of chambers volumes (Eqs. 18, 19). The above predictive model contains incremental equation of motion, which includes differences between actual acceleration and pressure values, and the values at the beginning of the control step. Moreover, it contains specific form of the energy balance equation.

The predictive model is used in three different manners:

- for simulation of the system response for arbitrarily assumed change of valve opening: Straightforward Dynamics Prediction (SDP),

- for computation of the valve opening corresponding to assumed kinematics of the system: Inverse Dynamics Prediction (IDP) and its generalization (GIDP).

- for computation of the optimal value of constant valve opening: Sub-Optimal Dynamics Prediction (SODP).

The proposed implementations of the IPC are reflected by the following control strategies:

1. Optimal control strategy: pneumatic force and impacting object deceleration provide minimization of the path-tracking error by exact solution of the variational problem, which is found in the class of valve openings changed continuously at single control step.

2. Control strategy with constant valve opening: pneumatic force and impacting object deceleration ensure minimal value of the path-tracking error by the solution found in the class of constant valve openings at a single control step.

3. Control strategy with assumed kinematics: pneumatic force and impacting object deceleration ensure minimal value of path-tracking error by the solution found in the class of assumed functions describing change of deceleration within a control step.

The optimization problems which are solved after identification of the system and excitation parameters are summarized in Table 1. All these alternatives of 
Table 1 Summary of optimization problems solved within different implementations of the IPC

\begin{tabular}{ll}
\hline Optimal control strategy & Find $\mathrm{A}_{v}^{\text {opt }}(\mathrm{t})=\arg \min \int_{\mathrm{t}_{\mathrm{i}}}^{\mathrm{t}_{\mathrm{i}}+\Delta \mathrm{t}}\left(\ddot{\mathrm{u}}\left(\mathrm{A}_{v}(\mathrm{t}), \mathrm{t}\right)+\frac{\mathrm{u}\left(\mathrm{t}_{\mathrm{i}}\right)^{2}}{2\left(\mathrm{~d}-\mathrm{u}\left(\mathrm{t}_{\mathrm{i}}\right)\right)}\right)^{2} \mathrm{dt}$ \\
Control strategy with constant valve opening & Find $\mathrm{A}_{v}^{\text {opt }}=\arg \min \int_{\mathrm{t}_{\mathrm{i}}}^{\mathrm{t}_{\mathrm{i}}+\Delta \mathrm{t}}\left(\ddot{\mathrm{u}}\left(\mathrm{A}_{v}^{\text {const. }}, \mathrm{t}\right)+\frac{\dot{\mathrm{u}}\left(\mathrm{t}_{\mathrm{i}}\right)^{2}}{2\left(\mathrm{~d}-\mathrm{u}\left(\mathrm{t}_{\mathrm{i}}\right)\right)}\right)^{2} \mathrm{dt}$ \\
Control strategy with assumed kinematics & Find $\boldsymbol{\beta}^{\text {opt }}=\arg \min \int_{\mathrm{t}_{\mathrm{i}}}^{\mathrm{t}_{\mathrm{i}}+\Delta \mathrm{t}}\left(\ddot{\mathrm{u}}(\boldsymbol{\beta}, \mathrm{t})+\frac{\dot{\mathrm{u}}\left(\mathrm{t}_{\mathrm{i}}\right)^{2}}{2\left(\mathrm{~d}-\mathrm{u}\left(\mathrm{t}_{\mathrm{i}}\right)\right)}\right)^{2} \mathrm{dt}$ \\
& where $\boldsymbol{\beta}$ - vector of coefficients defining function ü(t) at the control step $\Delta \mathrm{t}$
\end{tabular}

the Identification-based Predictive Control are derived in the following sections.

\section{Identification-based Predictive Control: optimal control strategy}

The optimal control strategy is aimed at finding exact unconstrained solution of the kinematics-based pathtracking (Eq. 37) formulated at a single control step. The solution assumes continuous change of valve opening in the considered time period $\left[\mathrm{t}_{\mathrm{i}}, \mathrm{t}_{\mathrm{i}}+\Delta \mathrm{t}\right]$.

\subsection{Theoretical solution at a single control step}

The objective of the formulated control problem is to find continuous function defining change of valve opening which minimizes path-tracking error:

Find $\mathrm{A}_{v}^{\mathrm{opt}}(\mathrm{t})=\arg \min \int_{\mathrm{t}_{\mathrm{i}}}^{\mathrm{t}_{\mathrm{i}}+\Delta \mathrm{t}}\left(\ddot{\mathrm{u}}\left(\mathrm{A}_{v}(\mathrm{t}), \mathrm{t}\right)+\frac{\dot{\mathrm{u}}\left(\mathrm{t}_{\mathrm{i}}\right)^{2}}{2\left(\mathrm{~d}-\mathrm{u}\left(\mathrm{t}_{\mathrm{i}}\right)\right)}\right)^{2} \mathrm{dt}$

Although finding constrained solution of the above problem requires methods of variational calculus, the unconstrained solutions can be easily found in intuitive manner. Theoretical solution, which does not take into account constitutive equation of the fluid, results from comparing the integrand of Eq. 45 to zero and reads:

$\ddot{\mathrm{u}}^{\text {opt }}\left(\mathrm{A}_{v}(\mathrm{t}), \mathrm{t}\right)=-\frac{\dot{\mathrm{u}}\left(\mathrm{t}_{\mathrm{i}}\right)^{2}}{2\left(\mathrm{~d}-\mathrm{u}\left(\mathrm{t}_{\mathrm{i}}\right)\right)}$

Thus, at a single control step the impacting object deceleration should remain constant and equal to optimal value calculated at the initial time instant. Between the control steps it should suffer a jump, whose direction depends on the sign of the difference between the actually optimal and actual value of deceleration. In the case of pneumatic absorber, the sudden change of impacting object's deceleration to the optimal value is impossible due to physical properties of the medium and limitation of maximal opening of the valve. The optimal change of piston deceleration can be deduced using the Eq. 46, which implies that:

- it should remain constant, $\ddot{\mathrm{u}}\left(\mathrm{A}_{v}(\mathrm{t}), \mathrm{t}\right)=\ddot{\mathrm{u}}\left(\mathrm{t}_{\mathrm{i}}\right)$,

- it should increase at maximal rate (valve fully closed) and after possible reaching of the optimal level should remain constant,

- it should decrease at maximal rate (valve fully open), and after possible reaching of the optimal level should remain constant,

in the cases of actual value of deceleration being equal, smaller and larger than the optimal one, respectivelly. Let us notice that in the first case the required valve opening $\mathrm{A}_{v}(\mathrm{t})$ will not be constant but it will continuously change over time. In the non-degenerated second and third case, the optimal value of deceleration might be reached or not during a single control step depending on the actual state of the system. The optimal changes of deceleration corresponding to two possible non-trivial cases are presented graphically in Fig. 4a and b, respectively.

\subsection{The control strategy}

The optimal two-stage control strategy includes three subsequent steps:

1. The identification step: identification of the difference of external and disturbance force in order to obtain predictive model of the system for the considered control step. 
Fig. 4 Optimal change of deceleration during a single control step: a the case of too low value of initial deceleration, $\mathbf{b}$ the case of too high value of initial deceleration

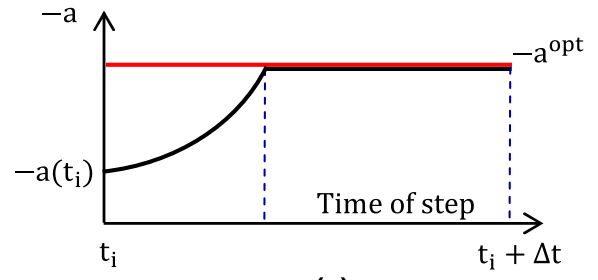

(a)

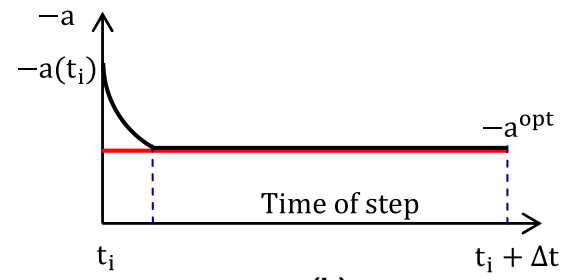

(b)
2. The prediction step: application of the SDP to simulate the system response with fully open or closed valve and check whether the optimal value of deceleration is reached within the actual control step.

3. The control determination step: application of the IDP to compute change of valve opening during the second part of the control step when deceleration remains constant.

The identification step starts with identification of the difference of external and disturbance force at each subsequent control step and it is based on Eq. 38 . Determined difference of external and disturbance forces is used to obtain specific forms of the equation of motion and equation of global energy balance used in the predictive model, which is applied in both the prediction step and the control determination step.

The prediction step follows the first stage of theoretical solution of the variational problem formulated for a single control step (Sect. 4.1). The predictive model is applied to conduct the Straightforward Dynamics Prediction (SDP) and simulate response of the system with fully closed or fully open valve. The SDP procedure utilizes system of equations governing absorber response at a single control step (Eqs. 3944), which includes the valve flow equation assuming minimal or maximal valve opening:

$\mathrm{A}_{v}(\mathrm{t})= \begin{cases}\mathrm{A}_{v}^{\min } & \text { for } \ddot{\mathrm{u}}\left(\mathrm{t}_{\mathrm{i}}\right)>\mathrm{a}_{\mathrm{opt}}\left(\mathrm{t}_{\mathrm{i}}\right) \\ \mathrm{A}_{v}^{\max } & \text { for } \ddot{\mathrm{u}}\left(\mathrm{t}_{\mathrm{i}}\right)<\mathrm{a}_{\text {opt }}\left(\mathrm{t}_{\mathrm{i}}\right)\end{cases}$

The simulation of the system response is conducted for the first part of the control step, i.e. until the time instant when predicted deceleration reaches optimal value defined by the r.h.s. of Eq. 46 .

The control determination step starts when piston deceleration achieves optimal value and it is conducted until the end of the control step. The predictive model is applied to perform the Inverse Dynamics Prediction (IDP) and compute change of valve opening $\mathrm{A}_{v}(\mathrm{t})$ which provides constant deceleration of the impacting object during the second part of the control step. The IDP procedure assumes constant value of the difference of external and disturbance force and it is conducted using the same predictive model as the SDP procedure applied during prediction step. However, in the IDP the valve opening is considered as time dependent unknown, while the system of governing equations is enriched by the definition of system kinematics resulting from the condition of piston deceleration being constant:

$\ddot{\mathrm{u}}^{\mathrm{opt}}=\ddot{\mathrm{u}}\left(\mathrm{t}_{\mathrm{i}}^{\mathrm{cc}}\right)$

$\mathrm{v}^{\mathrm{opt}}=\ddot{\mathrm{u}}\left(\mathrm{t}_{\mathrm{i}}^{\mathrm{cc}}\right)\left(\mathrm{t}-\mathrm{t}_{\mathrm{i}}^{\mathrm{cc}}\right)+\mathrm{v}\left(\mathrm{t}_{\mathrm{i}}^{\mathrm{cc}}\right)$

$\mathrm{u}^{\mathrm{opt}}=\frac{1}{2} \ddot{\mathrm{u}}\left(\mathrm{t}_{\mathrm{i}}^{\mathrm{cc}}\right)\left(\mathrm{t}-\mathrm{t}_{\mathrm{i}}^{\mathrm{cc}}\right)^{2}+\mathrm{v}\left(\mathrm{t}_{\mathrm{i}}^{\mathrm{cc}}\right)\left(\mathrm{t}-\mathrm{t}_{\mathrm{i}}^{\mathrm{cc}}\right)+\mathrm{u}\left(\mathrm{t}_{\mathrm{i}}^{\mathrm{cc}}\right)$

where $t_{i}^{c c}$ denotes the end of the first part of the control step (time of control change). The above kinematic relations allow to determine changes of chambers volumes $\left(\mathrm{V}_{1}^{\mathrm{opt}}\right.$ and $\left.\mathrm{V}_{2}^{\mathrm{opt}}\right)$ during the second stage of the process. As a result, selected equations of the predictive model (Eqs. 39, 41, 42, 43) can be rewritten as a system of algebraic equations:

$$
\begin{aligned}
& \mathrm{p}_{2} \mathrm{~A}_{2}-\mathrm{p}_{1} \mathrm{~A}_{1}=\mathrm{p}_{2}\left(\mathrm{t}_{\mathrm{i}}^{\mathrm{cc}}\right) \mathrm{A}_{2}-\mathrm{p}_{1}\left(\mathrm{t}_{\mathrm{i}}^{\mathrm{cc}}\right) \mathrm{A}_{1} \\
& \mathrm{~m}_{1}+\mathrm{m}_{2}=\mathrm{m} \\
& \left(\mathrm{p}_{2}\left(\mathrm{t}_{\mathrm{i}}^{\mathrm{cc}}\right) \mathrm{A}_{2}-\mathrm{p}_{1}\left(\mathrm{t}_{\mathrm{i}}^{\mathrm{cc}}\right) \mathrm{A}_{1}\right)\left(\mathrm{u}^{\mathrm{opt}}(\mathrm{t})-\mathrm{u}\left(\mathrm{t}_{\mathrm{i}}^{\mathrm{cc}}\right)\right) \\
& =\frac{\mathrm{p}_{1} \mathrm{~V}_{1}^{\mathrm{opt}}}{\kappa-1}+\frac{\mathrm{p}_{2} \mathrm{~V}_{2}^{\mathrm{opt}}}{\kappa-1}-\frac{\mathrm{p}_{1}\left(\mathrm{t}_{\mathrm{i}}^{\mathrm{cc}}\right) \mathrm{V}_{1}\left(\mathrm{t}_{\mathrm{i}}^{\mathrm{cc}}\right)}{\kappa-1}-\frac{\mathrm{p}_{2}\left(\mathrm{t}_{\mathrm{i}}^{\mathrm{cc}}\right) \mathrm{V}_{2}\left(\mathrm{t}_{\mathrm{i}}^{\mathrm{cc}}\right)}{\kappa-1}
\end{aligned}
$$


$\frac{\mathrm{p}_{2}\left(\mathrm{~V}_{2}^{\mathrm{opt}}\right)^{\kappa}}{\mathrm{m}_{2}^{\kappa}}=\frac{\mathrm{p}_{2}\left(\mathrm{t}_{\mathrm{i}}^{\mathrm{cc}}\right) \mathrm{V}_{2}\left(\mathrm{t}_{\mathrm{i}}^{\mathrm{cc}}\right)^{\kappa}}{\mathrm{m}_{2}\left(\mathrm{t}_{\mathrm{i}}^{\mathrm{cc}}\right)^{\kappa}}$

The Eqs. 49 indicates that pneumatic force generated by the absorber remains constant and this fact is also used in the equation governing global energy balance Eq. 51. The set of Eqs. 49-52 contains four unknowns: $\mathrm{m}_{1}, \mathrm{~m}_{2}, \mathrm{p}_{1}, \mathrm{p}_{2}$ and can be solved analytically in order to determine the change of system state during the second part of the control step. Further, the IDP procedure utilizes mass balance equation combined with the equation of the valve flow in order to calculate required change of valve opening:

$\mathrm{A}_{v}^{\mathrm{opt}}(\mathrm{t})=-\dot{\mathrm{m}}_{2}\left[\sqrt{\frac{2 \kappa}{\kappa-1}\left(\mathrm{q}^{\frac{2}{\kappa}}-\mathrm{q}^{\frac{\kappa+1}{\kappa}}\right)} \frac{\mathrm{p}_{2}}{\sqrt{\mathrm{RT}_{2}}}\right]^{-1}$

It is assumed that valve chosen such that $\mathrm{A}_{v}^{\mathrm{opt}}(\mathrm{t}) \leq \mathrm{A}_{v}^{\mathrm{max}}$ and realization of the process is always possible. Let us note that above determined valve opening will not provide exactly constant value of impacting object deceleration and pneumatic force in real system since, in contrast to predictive model, the dynamics of the real system includes changing in time external and disturbance forces. However, the analysis concerns only a single control step of relatively short duration so the change of external and disturbance force as well as corresponding change of the impacting object deceleration is not expected to be significant. More sophisticated control strategy can utilize prediction of the future value of external force based on identification of its derivative at the beginning of the control step.

The above two-stage procedure composed of identification step, prediction step and control determination step is repeated at the following control steps of the analysis. The above derived optimal two-stage control strategy possess three basic features characterizing self-adaptive systems: (i) elimination of the requirement of a priori knowledge of dynamic excitation, (ii) robust operation in case of subsequent impacts arising during the process, (iii) robustness to disturbances such as additional forces generated in the absorber (e.g. friction or elastic forces).
4.3 The architecture of the control system

The above developed control strategy can be presented in the form of block diagram of the control system. The presented diagram of the Identification-based Predictive Control system follows a general scheme of self-adaptive control systems [36], but it is more detailed and complicated. Except the classical measurement (sensors) block and valve control block, the proposed control system includes several additional blocks located inside the feedback control loop. As shown in Fig. 5, the scheme of the control system corresponding to optimal two-stage control method contains the following function blocks inside the feedback control loop:

1. the kinematic block, which calculates actual optimal value of impacting object deceleration and determines whether deceleration should increase or decrease during the first part of the control step (data for the prediction block);

2. the identification block, which identifies the actual value of difference between the external and disturbance force (data for the prediction and correction blocks);

3. the prediction block, which allows to predict response of the system with fully closed or fully open valve during a single control step and determines whether the optimal value of impacting object deceleration is reached within the actual control step (data for correction block);

4. the correction block activated when the condition of obtaining optimal deceleration satisfied, which determines time-dependent change of the valve opening providing constant value of deceleration during the second part of the control step.

The kinematic block, identification block and prediction block are always present inside the feedback control loop. In turn, the correction block is connected by the switch and it can be either activated or not, depending on the results obtained from the prediction block. 


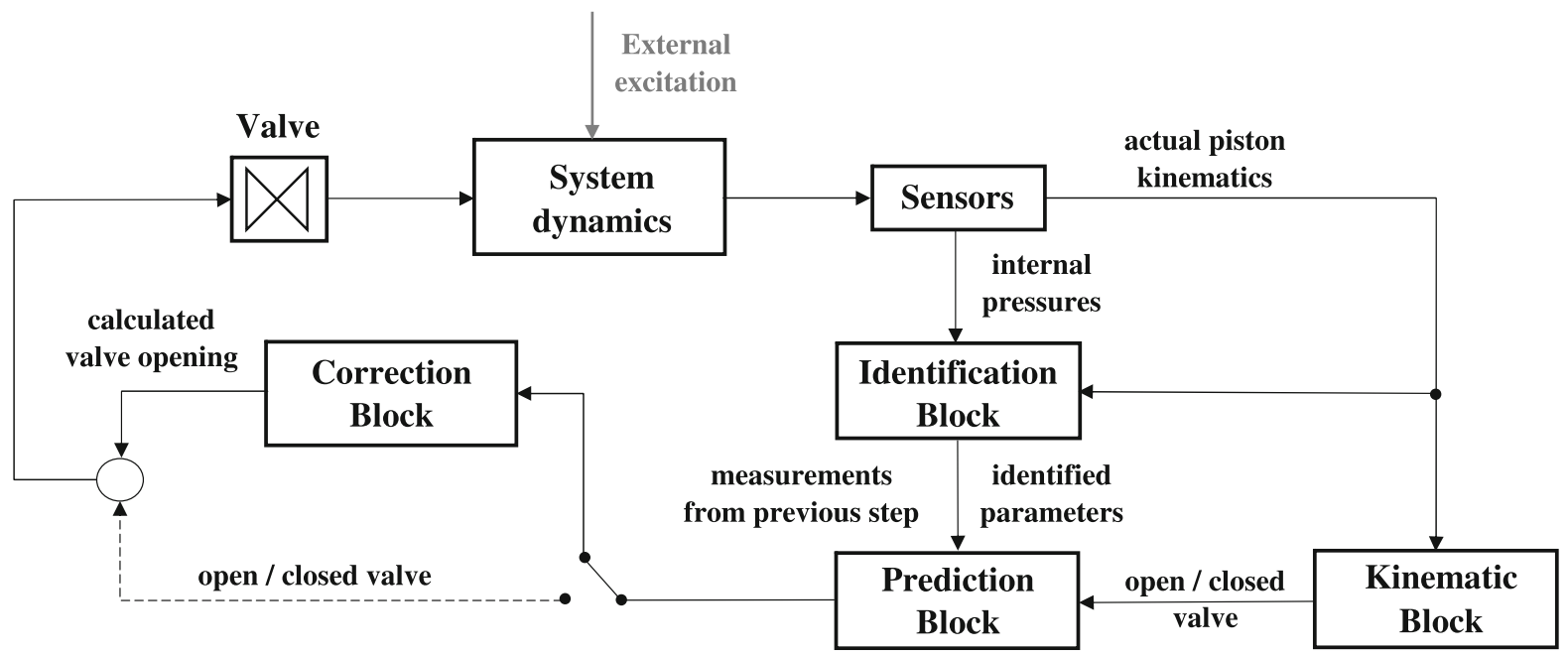

Fig. 5 Scheme of the control system corresponding to optimal two-stage control strategy

\section{Identification-based predictive control: strategy with constant valve opening}

The second control strategy is aimed at approximation of the optimal control of valve opening by applying optimized constant opening of the valve during each control step. In contrast to optimal control strategy considered in Sect. 4 each control step of this strategy does not contain two separate stages at which valve opening is determined in different manner. In turn, the constant valve opening is selected once for the entire control step.

\subsection{The control strategy}

The derivation of the standard version of control strategy with constant valve opening contains three subsequent steps:

1. The identification step: identification of the difference of external and disturbance force in order to obtain predictive model of the system.

2 . The prediction step: application of straightforward dynamics prediction (SDP) to simulate system response with fully open or fully closed valve.

3. The control determination step: application of sub-optimal dynamics prediction (SODP) to compute optimal value of constant valve opening.
The identification step and prediction step are conducted in the same manner as in the case of optimal two-stage control strategy.

The control determination step is conducted for the entire period of the considered control step and it utilizes the Sub-Optimal Dynamics Prediction (SODP). The SODP procedure is based on predictive model defined by Eqs. 39-44, which is applied for computation of the expected system response. The substantial difference is that the valve opening is assumed as:

$\mathrm{A}_{v}(\mathrm{t})=\mathrm{A}_{v}^{\text {const. }}$

where $\mathrm{A}_{v}^{\text {const. }}$ is unknown constant area of the valve opening. The SODP procedure is aimed at finding optimal value of constant valve opening $\mathrm{A}_{v}^{\mathrm{opt}}$, which provides minimization of the integral discrepancy between predicted deceleration and its actual optimal value. The corresponding optimality condition takes the form:

$\mathrm{A}_{v}^{\text {opt }}=\arg \min \int_{\mathrm{t}_{\mathrm{i}}}^{\mathrm{t}_{\mathrm{i}}+\Delta \mathrm{t}}\left(\ddot{\mathrm{u}}\left(\mathrm{A}_{v}^{\text {const. }}, \mathrm{t}\right)+\frac{\dot{\mathrm{u}}\left(\mathrm{t}_{\mathrm{i}}\right)^{2}}{2\left(\mathrm{~d}-\mathrm{u}\left(\mathrm{t}_{\mathrm{i}}\right)\right)}\right)^{2} \mathrm{dt}$

Repeated numerical solving of the system of nonlinear differential equations (Eqs. 39-44) in order to find $\mathrm{A}_{v}^{\mathrm{opt}}$ 
satisfying condition given by Eq. 55 is computationally relatively expensive, so simplification and speed up of the entire procedure is recommended in practical applications. This can be achieved by: (i) proper choice of the initial value of constant valve opening for the optimization procedure, (ii) simplified solution of the governing equations (Eqs. 39-44) based on linearization of the response at a single control step.

The initial value of constant valve opening for the optimization procedure can be determined using the inverse dynamics approach, which enables computation of the valve opening providing constant deceleration of the impacting object during a single control step, cf. Eqs. 49-52. Such initial value can be assumed either as initial or average value of optimal timedependent valve opening determined from IDP:

$$
\begin{aligned}
& \mathrm{A}_{v}^{\mathrm{ini}}\left(\mathrm{t}_{\mathrm{i}}\right)=\mathrm{A}_{v}^{\mathrm{opt}}\left(\mathrm{t}_{\mathrm{i}}\right) \quad \text { or } \\
& \mathrm{A}_{v}^{\mathrm{ini}}\left(\mathrm{t}_{\mathrm{i}}\right)=\frac{1}{2}\left[\mathrm{~A}_{v}^{\mathrm{opt}}\left(\mathrm{t}_{\mathrm{i}}\right)+\mathrm{A}_{v}^{\mathrm{opt}}\left(\mathrm{t}_{\mathrm{i}}+\Delta \mathrm{t}\right)\right]
\end{aligned}
$$

where $\mathrm{A}_{v}^{\mathrm{opt}}\left(\mathrm{t}_{\mathrm{i}}\right)$ and $\mathrm{A}_{v}^{\mathrm{opt}}\left(\mathrm{t}_{\mathrm{i}}+\Delta \mathrm{t}\right)$ can be determined from Eq. 53. Since the definite integral defined by Eq. 55 is convex in terms of constant valve opening and the initial point is correctly chosen, the optimization problem can be solved using standard gradientbased methods and converges fast.

The second approach assumes standard linearization of the Eqs. 39-44 at a single control step with respect to selected state variables and analytical solution of the system of linearized differential equations for constant valve opening. Alternatively, the original system of differential equations governing the problem can be solved using forward Euler method with only one step of integration applied at each control step $\left[\mathrm{t}_{\mathrm{i}}, \mathrm{t}_{\mathrm{i}}+\Delta \mathrm{t}\right]$. Both above methods result in analytical formula defining the value of impacting object deceleration in terms of system state at the beginning of the control step, applied constant valve opening $\mathrm{A}_{v}^{\text {const. }}$ and time of the process:

$$
\begin{aligned}
\ddot{\mathrm{u}}\left(\mathrm{A}_{v}^{\text {const. }}, \mathrm{t}\right)= & \mathrm{f}\left(\mathrm{p}_{1}\left(\mathrm{t}_{\mathrm{i}}\right), \mathrm{p}_{2}\left(\mathrm{t}_{\mathrm{i}}\right), \mathrm{m}_{1}\left(\mathrm{t}_{\mathrm{i}}\right), \mathrm{m}_{2}\left(\mathrm{t}_{\mathrm{i}}\right), \mathrm{u}\left(\mathrm{t}_{\mathrm{i}}\right),\right. \\
& \left.\mathrm{v}\left(\mathrm{t}_{\mathrm{i}}\right), \mathrm{a}\left(\mathrm{t}_{\mathrm{i}}\right), \mathrm{A}_{v}^{\text {const. }}, \mathrm{t}\right)
\end{aligned}
$$

where f indicates known analytical function. Providing that the version of thermodynamic energy balance equations is properly chosen, the formula defining change of acceleration in a single step is relatively simple and can be integrated analytically in time to obtain definite integral of the r.h.s. of Eq. 55 in the form of analytical formula $\mathrm{I}=\mathrm{I}\left(\mathrm{A}_{v}^{\text {const. }}\right)$. This allows to simplify the original optimization problem to the simple problem:

$\mathrm{A}_{v}^{\text {opt }}=\arg \min \mathrm{I}\left(\mathrm{A}_{v}^{\text {const. }}\right)$

which can be solved using standard minimization conditions involving zero value of the first and positive value of the second derivative with respect to $\mathrm{A}_{v}^{\text {const. }}$.

\subsection{Alternative version of control strategy with constant valve opening}

The above introduced control strategy with constant valve opening can be superseded by its alternative version, in which the prediction steps are entirely eliminated. The alternative version of control strategy with constant valve opening involves the following steps:

1. The identification step identification of the difference of external and disturbance force.

2. The control determination step using the SODP in order to determine the optimal constant valve opening for each control step using predictive model of the system.

In fact, in the standard version of the control strategy with constant valve opening the presence of the prediction steps was aimed at identifying the control steps at which specific non-extreme constant value of valve opening does not have to be calculated and control determination step does not have to be executed. Such approach was justified because the standard SDP procedure applied in prediction step requires only straightforward simulation of the system response, while SODP procedure applied in control determination step requires solution of the inverse problem. The control determination steps were associated with higher numerical cost than prediction steps and their elimination allowed for reducing the global computational cost.

However, linearization of the predictive model results in analytical formula defining change of acceleration at a single control step (Eq. 57) and possibility of finding optimal $\mathrm{A}_{v}^{\mathrm{opt}}$ in a single iteration 
(Eq. 58). Thus, the numerical cost of the control determination step is significantly reduced and it is similar to the cost of the prediction step. Consequently, the prediction steps can be replaced by control determination steps without significant increase of total computational cost. Such approach allows for simplification and unification of the entire control procedure. On the other hand, its disadvantage is that decision about application of fully closed or opened valve is burdened with an error resulting from linearization of the predictive model.

The above derived two versions of the control strategy with constant valve opening possess the basic features of the optimal two-stage strategy, including elimination of the requirement of a priori knowledge of dynamic excitation, robust operation in case of subsequent impacts and robustness to disturbances. As a result of the application of the SOPD assuming constant valve opening, the single-stage methods are expected to lead to slightly worse solution of the original variational problem than optimal two-stage methods. However, the single-stage methods are expected to require less intensive control actions with globally more smooth change of valve opening and only small jumps between the control steps.

\subsection{Architecture of the control system}

The scheme of the control system corresponding to control strategy with constant valve opening in its standard version (with prediction and control determination steps) is identical as the scheme of the control system corresponding to optimal two-stage method, presented in Fig. 5. The main difference between these two control systems is different realization of the correction block (SODP vs. IDP) and different time period for which the correction is introduced (the entire period of the control step vs. the second part of control step).

In contrast, the scheme of the control system corresponding to alternative version (without prediction step) includes the following control blocks inside the feedback control loop (Fig. 6):

1. the kinematic block, which calculates actual optimal value of impacting object deceleration (data for valve opening computation block);
2. the identification block, which determines the actual value of difference between the external and disturbance force (data for valve opening computation block);

3. the valve opening computation block, which determines constant valve opening providing minimal deviation of deceleration from the optimal value at considered control step.

Let us note that all above blocks are always present in the discussed version of the system and in contrast to the system presented in Fig. 5 the switched connection between two final blocks of the system does not appear.

\section{Identification-based predictive control: control strategy with assumed kinematics}

The last developed control method is aimed at approximation of the optimal two-stage control strategy by assuming arbitrary kinematics of the impacting object at each control step. The method combines the advantages of previously developed approaches: employment of the IDP introduced within the optimal two-stage strategy and elimination of prediction step as in the strategy with constant valve opening.

\subsection{The control strategy}

Derivation of the control strategy with assumed kinematics includes two subsequent steps:

1. The identification step: identification of the difference of external and disturbance force in order to obtain predictive model of the system at considered control step.

2. The control determination step: assumption of the arbitrary function describing the change of acceleration at a single control step, optimization its parameters using kinematics-based formulation and determination of the corresponding change of valve opening using IDP.

The key concept of the current version of the control determination step is assumption of the arbitrary type of function $\ddot{u}(\boldsymbol{\beta}, \mathrm{t})$ defining change of impacting object's deceleration during a single control 


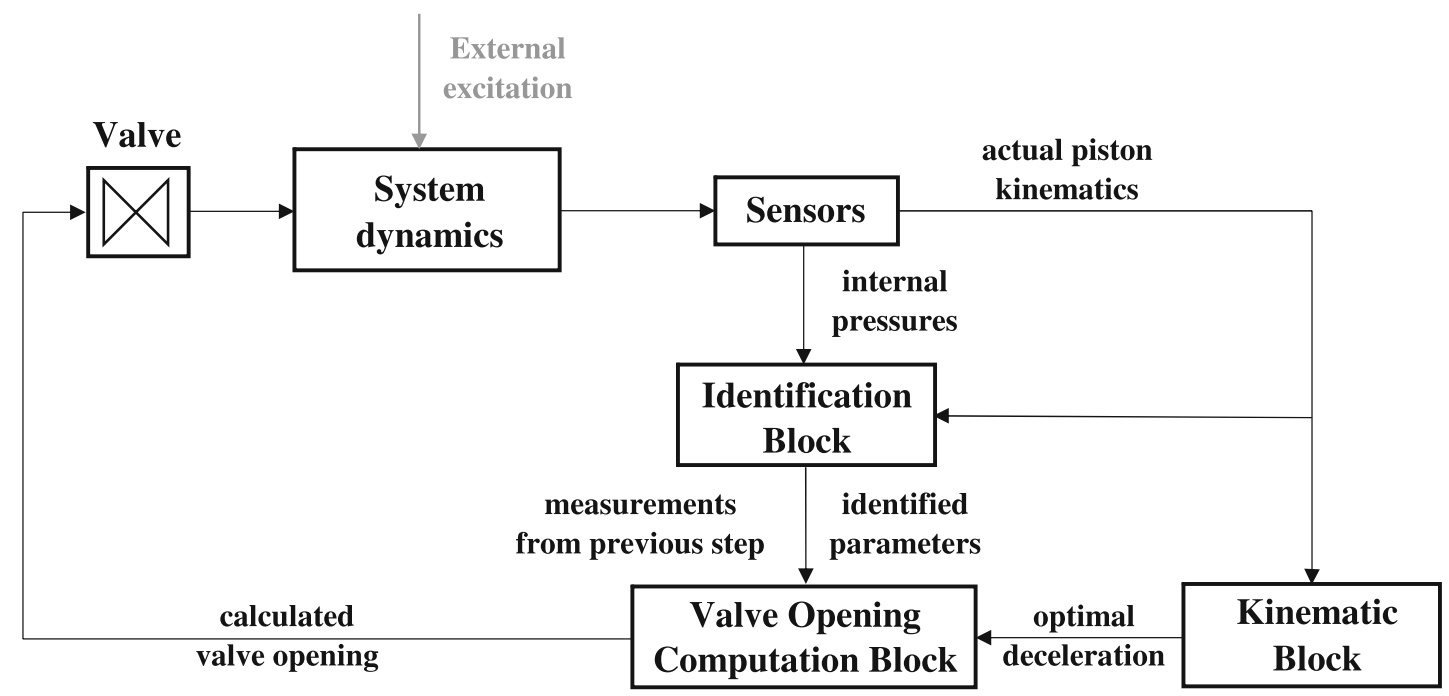

Fig. 6 Scheme of the control system corresponding to control strategy with constant valve opening

step, where $\boldsymbol{\beta}$ is the vector of unknown parameters calculated for actual time step. The optimization procedure is aimed at finding values of parameters which provide minimization of the integral discrepancy between assumed deceleration and its actual optimal value. The corresponding optimality condition takes the form:

$\boldsymbol{\beta}^{\text {opt }}=\arg \min \int_{\mathrm{t}_{\mathrm{i}}}^{\mathrm{t}_{\mathrm{i}}+\Delta \mathrm{t}}\left(\ddot{\mathrm{u}}(\boldsymbol{\beta}, \mathrm{t})+\frac{\dot{\mathrm{u}}\left(\mathrm{t}_{\mathrm{i}}\right)^{2}}{2\left(\mathrm{~d}-\mathrm{u}\left(\mathrm{t}_{\mathrm{i}}\right)\right)}\right)^{2} \mathrm{dt}$

In the second step, the determined deceleration path at a single control step is tracked using time-dependent change of the valve opening, which is computed using the predictive model of the system. Since a wide class of functions describing change of impacting object's deceleration can be assumed (e.g. linear, quadratic or exponential) the method yields a large class of solutions of the considered control problem.

In the simplest version of the method, deceleration of the impacting object is assumed to change linearly between the actual and the final value. Assumption of the continuity of deceleration at the initial time instant allows to define linear function describing change of deceleration in terms of only one unknown parameter being the value of acceleration at the end of the control step $\beta=\ddot{\mathrm{u}}\left(\mathrm{t}_{\mathrm{i}}+\Delta \mathrm{t}\right)$ : $\ddot{\mathrm{u}}(\beta, \mathrm{t})=\ddot{\mathrm{u}}\left(\mathrm{t}_{\mathrm{i}}\right)+\frac{\beta-\ddot{\mathrm{u}}\left(\mathrm{t}_{\mathrm{i}}\right)}{\Delta \mathrm{t}}\left(\mathrm{t}-\mathrm{t}_{\mathrm{i}}\right)$

In such case the optimization problem takes the form:

$$
\begin{aligned}
& \ddot{\mathrm{u}}^{\text {opt }}\left(\mathrm{t}_{\mathrm{i}}+\Delta \mathrm{t}\right)=\arg \min \int_{\mathrm{t}_{\mathrm{i}}}^{\mathrm{t}_{\mathrm{i}}+\Delta \mathrm{t}}\left(\ddot{\mathrm{u}}\left(\mathrm{t}_{\mathrm{i}}\right)+\frac{\beta-\ddot{\mathrm{u}}\left(\mathrm{t}_{\mathrm{i}}\right)}{\Delta \mathrm{t}}\left(\mathrm{t}-\mathrm{t}_{\mathrm{i}}\right)\right. \\
& \left.+\frac{\dot{\mathrm{u}}\left(\mathrm{t}_{\mathrm{i}}\right)^{2}}{2\left(\mathrm{~d}-\mathrm{u}\left(\mathrm{t}_{\mathrm{i}}\right)\right)}\right)^{2} \mathrm{dt}
\end{aligned}
$$

The above problem is convex and the solution can be obtained from standard differential conditions involving zero value of the first derivative and positive value of the second derivative with respect to $\beta$. The solution reads:

$\beta^{\text {opt }}=\ddot{\mathrm{u}}^{\text {opt }}\left(\mathrm{t}_{\mathrm{i}}+\Delta \mathrm{t}\right)=-\frac{\ddot{\mathrm{u}}\left(\mathrm{t}_{\mathrm{i}}\right)}{2}+\frac{3}{4} \frac{\dot{\mathrm{u}}\left(\mathrm{t}_{\mathrm{i}}\right)^{2}}{\left(\mathrm{~d}-\mathrm{u}\left(\mathrm{t}_{\mathrm{i}}\right)\right)}$

and indicates that discrepancy of impacting object's deceleration from the optimal value changes sign and its value decreases twice at each control step. This causes that actual deceleration is shifted towards the optimal deceleration determined at the end of each control step, and improves convergence of the entire process (indicated by zero value of integrand in Eq. 61). The knowledge of initial conditions at the beginning of the control step and the optimal final 
value of deceleration allows to determine the desired change of deceleration, velocity and displacement of the impacting object during the control step:

$$
\begin{aligned}
\ddot{\mathrm{u}}^{\text {opt }}= & \ddot{\mathrm{u}}\left(\mathrm{t}_{\mathrm{i}}\right)+\frac{\ddot{\mathrm{u}}^{\mathrm{opt}}\left(\mathrm{t}_{\mathrm{i}}+\Delta \mathrm{t}\right)-\ddot{\mathrm{u}}\left(\mathrm{t}_{\mathrm{i}}\right)}{\Delta \mathrm{t}}\left(\mathrm{t}-\mathrm{t}_{\mathrm{i}}\right) \\
\mathrm{v}^{\mathrm{opt}}= & \frac{1}{2} \frac{\ddot{\mathrm{u}}^{\text {opt }}\left(\mathrm{t}_{\mathrm{i}}+\Delta \mathrm{t}\right)-\ddot{\mathrm{u}}\left(\mathrm{t}_{\mathrm{i}}\right)}{\Delta \mathrm{t}}\left(\mathrm{t}-\mathrm{t}_{\mathrm{i}}\right)^{2}+\ddot{\mathrm{u}}\left(\mathrm{t}_{\mathrm{i}}\right)\left(\mathrm{t}-\mathrm{t}_{\mathrm{i}}\right) \\
& +\mathrm{v}\left(\mathrm{t}_{\mathrm{i}}\right) \\
\mathrm{u}^{\mathrm{opt}}= & \frac{1}{6} \frac{\ddot{\mathrm{u}}^{\text {opt }}\left(\mathrm{t}_{\mathrm{i}}+\Delta \mathrm{t}\right)-\ddot{\mathrm{u}}\left(\mathrm{t}_{\mathrm{i}}\right)}{\Delta \mathrm{t}}\left(\mathrm{t}-\mathrm{t}_{\mathrm{i}}\right)^{3}+\frac{1}{2} \ddot{\mathrm{u}}\left(\mathrm{t}_{\mathrm{i}}\right)\left(\mathrm{t}-\mathrm{t}_{\mathrm{i}}\right)^{2} \\
& +\mathrm{v}\left(\mathrm{t}_{\mathrm{i}}\right)\left(\mathrm{t}-\mathrm{t}_{\mathrm{i}}\right)+\mathrm{u}\left(\mathrm{t}_{\mathrm{i}}\right)
\end{aligned}
$$

as well as the corresponding changes of chambers volumes $\left(\mathrm{V}_{1}^{\mathrm{opt}}\right.$ and $\left.\mathrm{V}_{2}^{\mathrm{opt}}\right)$. The full knowledge of the system kinematics allows to apply the concept of Generalized Inverse Dynamics Prediction and compute valve opening corresponding to non-constant impacting object deceleration. The system of equations used to determine change of thermodynamic parameters of gas is obtained by substitution of derived optimal kinematics into the predictive model of the system and reads:

$$
\begin{aligned}
& \mathrm{p}_{2} \mathrm{~A}_{2}-\mathrm{p}_{1} \mathrm{~A}_{1}=\mathrm{p}_{2}\left(\mathrm{t}_{\mathrm{i}}\right) \mathrm{A}_{2}-\mathrm{p}_{1}\left(\mathrm{t}_{\mathrm{i}}\right) \mathrm{A}_{1}-\mathrm{M}\left[\ddot{\mathrm{u}}^{\text {opt }}-\ddot{\mathrm{u}}\left(\mathrm{t}_{\mathrm{i}}\right)\right] \\
& \mathrm{m}_{1}+\mathrm{m}_{2}=\mathrm{m} \\
& \frac{1}{2} \mathrm{Mv}\left(\mathrm{t}_{\mathrm{i}}\right)^{2}-\frac{1}{2} \mathrm{M}\left(\mathrm{v}^{\mathrm{opt}}\right)^{2} \\
& +\left[\mathrm{Mu}\left(\mathrm{t}_{\mathrm{i}}\right)+\left(\mathrm{p}_{2}\left(\mathrm{t}_{\mathrm{i}}\right) \mathrm{A}_{2}-\mathrm{p}_{1}\left(\mathrm{t}_{\mathrm{i}}\right) \mathrm{A}_{1}\right)\right]\left(\mathrm{u}^{\text {opt }}-\mathrm{u}\left(\mathrm{t}_{\mathrm{i}}\right)\right) \\
& =\frac{\mathrm{p}_{1} \mathrm{~V}_{1}^{\text {opt }}}{\kappa-1}+\frac{\mathrm{p}_{2} \mathrm{~V}_{2}^{\text {opt }}}{\kappa-1}-\frac{\mathrm{p}_{1}\left(\mathrm{t}_{\mathrm{i}}\right) \mathrm{V}_{1}\left(\mathrm{t}_{\mathrm{i}}\right)}{\kappa-1} \\
& \quad-\frac{\mathrm{p}_{2}\left(\mathrm{t}_{\mathrm{i}}\right) \mathrm{V}_{2}\left(\mathrm{t}_{\mathrm{i}}\right)}{\kappa-1}
\end{aligned}
$$

$\frac{\mathrm{p}_{2}\left(\mathrm{~V}_{2}^{\mathrm{opt}}\right)^{\kappa}}{\mathrm{m}_{2}^{\kappa}}=\frac{\mathrm{p}_{2}\left(\mathrm{t}_{\mathrm{i}}^{\mathrm{cc}}\right) \mathrm{V}_{2}\left(\mathrm{t}_{\mathrm{i}}^{\mathrm{cc}}\right)^{\kappa}}{\mathrm{m}_{2}\left(\mathrm{t}_{\mathrm{i}}^{\mathrm{cc}}\right)^{\kappa}}$

The above system of algebraic equations describing GIDP has substantially different form than the corresponding system of equations describing standard IDP, which is used for maintaining constant value of deceleration (Eqs. 49-52). In particular, the equation describing change of pneumatic force includes additional term depending on the impacting object mass and its optimal deceleration, cf. the r.h.s. of Eqs. 49 and 66. Moreover, in equation of global energy balance the work done on gas by external forces is expressed in terms of impacting object mass and its optimal velocity, cf. the l.h.s. of Eqs. 51 and 68 . Although equations governing the GIDP are relatively complicated, the change of thermodynamic parameters of the fluid can be determined analytically. Further, the corresponding change of valve opening during the considered control step is calculated using valve flow equation (Eq. 53). The above described procedure, which is based on identification step and control determination step, is repeated for the subsequent control steps of the impact absorption process.

The above derived control method with assumed kinematics possess basic features of the optimal twostage methods including the lack of requirement of a priori knowledge of dynamic excitation, robust operation in the case of subsequent impacts and disturbances. As a result of the assumption of non-optimal, e.g. linear change of impacting object deceleration, the method is expected to lead to slightly worse solution of the original variational problem than optimal twostage method, but similar as the single-stage method with constant valve opening.

The single-stage method with assumed kinematics is expected to require control actions of comparable intensity as previously developed single-stage control methods with constant valve opening. Nevertheless, it will require continuous and smooth change of valve opening with modifications during a single control step, but without small jumps between the steps. Moreover, due to application of the IDP the numerical cost of the method is also expected to be comparable with the cost of the method with constant valve opening based on linearized predictive model. In general, the difference between the control strategy with constant valve opening and the control strategy with assumed kinematics is expected to be very subtle.

\subsection{Architecture of the control system}

The scheme of the control system corresponding to the control strategy with assumed kinematics is similar to previously introduced control scheme developed for the alternative version of the control strategy with 
constant valve opening (Fig. 6). It includes the following blocks inside the feedback control loop:

1. the kinematic block, which calculates actual optimal value of impacting object deceleration and assumes function describing the change of deceleration during the entire control step (data for valve opening computation block);

2. the identification block, which identifies either the actual value of difference between the external and disturbance force (data for valve opening computation block);

3. the valve opening computation block, which determines system kinematics ensuring minimal discrepancy of deceleration from the optimal value at considered control step and the corresponding valve opening.

Similarly as in the previous control scheme, all above mentioned blocks are always present and the connection by switch does not appear. The only difference in comparison to control scheme for constant valve opening is that the output from kinematic block is not only the information concerning actual optimal value of deceleration but also assumed function describing change of acceleration.

\section{Numerical study on performance of the identification-based predictive controllers}

The numerical verification of the operation of the developed Identification-based Predictive Control algorithms is conducted using double-chamber pneumatic shock-absorber under impact excitation caused by impact of a rigid object. The values of external and disturbance forces are assumed to be unknown. For the ease of comparisons, the dimensions of the shockabsorber, initial parameters of gas and parameters of the impact excitation are the same as in most of the examples discussed in previous papers of the authors (Table 2). In order to conduct clear analyses of system robustness two types of disturbances are considered: (i) linearly increasing elastic force with stiffness coefficient $\mathrm{k}=2000 \mathrm{~N} / \mathrm{m}$, (ii) linearly decreasing viscous force with damping coefficient $\mathrm{c}=40 \mathrm{Ns} / \mathrm{m}$, both acting at the entire stroke of the absorber. Separate simulations are conducted for three implementations of the IPC:

- Optimal two-stage control strategy,

- Strategy with constant valve opening,

- Control strategy with assumed kinematics.

The operation of all three systems is assessed under disturbance caused by elastic and viscous forces. The methods are thoroughly compared against each other in terms of efficiency and required control actions.

\subsection{Optimal control strategy}

The optimal two-stage control strategy was initially tested using impact excitation and unknown elastic disturbance force. The control strategy includes two distinct stages of valve control. In the first stage of the process the valve remains closed in order to obtain possibly fast increase of force generated by the absorber, up to the level required for dissipation of the entire impact energy. In the second stage of the process the intensive control actions with fast changes of valve opening are performed (Fig. 7a) in order to maintain approximately constant level of total force generated by the shock-absorber until the end of the stroke (Fig. 7b). During the first part of each control step the valve is fully opened in order to compensate the increase of total generated force caused by the increase of elastic disturbance force. In turn, during the second part of each control step the valve opening

Table 2 Parameters of the shock-absorber, the gas state, the impact loading and the disturbance assumed in presented numerical examples

\begin{tabular}{|c|c|c|c|c|c|c|c|c|c|}
\hline $\begin{array}{l}\mathrm{M} \\
{[\mathrm{kg}]}\end{array}$ & $\begin{array}{l}\mathrm{v}_{0} \\
{[\mathrm{~m} / \mathrm{s}]}\end{array}$ & Gas & $\begin{array}{l}\mathrm{R} \\
{[\mathrm{J} / \mathrm{kg} / \mathrm{K}]}\end{array}$ & $\begin{array}{l}\mathrm{p}_{1}^{0}, \mathrm{p}_{2}^{0} \\
{[\mathrm{kPa}]}\end{array}$ & $\begin{array}{l}\mathrm{T}_{1}^{0}, \mathrm{~T}_{2}^{0} \\
{[\mathrm{~K}]}\end{array}$ & $\begin{array}{l}\mathrm{V}_{1}^{0} \\
{\left[\mathrm{~cm}^{3}\right]}\end{array}$ & $\begin{array}{l}\mathrm{V}_{2}^{0} \\
{\left[\mathrm{~cm}^{3}\right]}\end{array}$ & $\begin{array}{l}\mathrm{h}_{1}^{0} \\
{[\mathrm{~mm}]}\end{array}$ & $\begin{array}{l}\mathrm{h}_{2}^{0} \\
{[\mathrm{~mm}]}\end{array}$ \\
\hline 5 & 5 & air & 287.69 & 300 & 293.15 & 7.54 & 118.12 & 6 & 94 \\
\hline
\end{tabular}




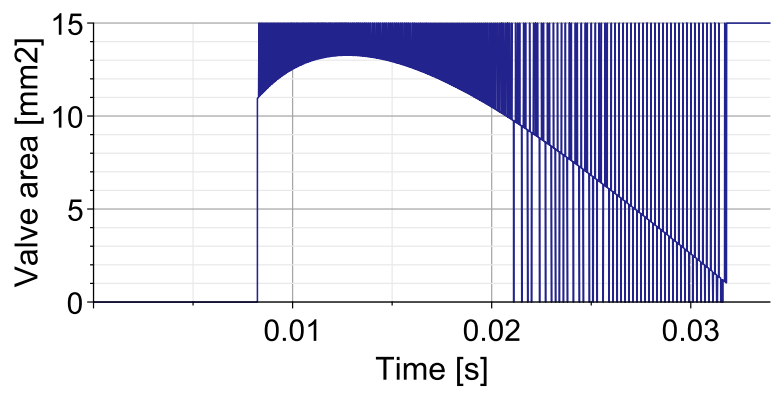

(a)

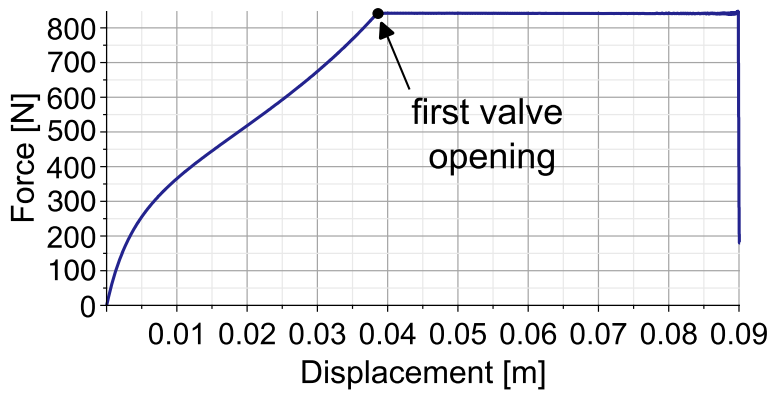

(b)

Fig. 7 Optimal two-stage control strategy, disturbance introduced by the elastic force: a change of valve opening area, $\mathbf{b}$ change of total force generated by the absorber

changes in time in order to maintain exactly constant value of force calculated using predictive model and approximately constant value of force in the real system. The optimal change of valve opening calculated during the second part of each control step creates the characteristic time-history of continuous phases of valve opening with the initial increase and gradual decease almost to zero value at the end of the process.

Let us note that at certain time instant of the process the valve is commutatively opened and closed at the consecutive control steps, which can be considered as sudden increase of the required control effort. This phenomenon is related to finite lengths of the time steps used in applied numerical integration procedure and the lengths of time periods when the valve opening has to remain extreme. The detailed explanation is as follows. At each consecutive control step the initial time period when the valve remains fully open gradually decreases due to reduction of piston velocity and corresponding reduction of pneumatic force increase, which is driven by change of chambers' volumes. Eventually, it drops below the length of the applied time integration step. Since the condition for finishing the initial stage of maximal valve opening is checked at the end of the time step the valve remains open for excessive amount of time, which results in drop of pneumatic force below the optimal level. Further, the non-optimal level of pneumatic force is maintained constant, but this effect is compensated by gradual increase of elastic disturbance force. As a result, at the end of the control step the total value of generated force is larger than the optimal one and at the valve is again fully opened at the beginning of the next control step. However, the effect of elastic force increase diminishes as the process of impact absorption proceeds due to decreasing velocity of the piston. Eventually, the optimal value of impacting object deceleration is not achieved until the end of the control step. As a result, the next control step commences with the stage when the valve is fully closed. The above effect of sudden increase of the control effort is also expected to appear in practical realization of the control system due to finite length of minimal time of valve opening.

The optimal two-stage control strategy was also investigated using in the case of unknown viscous disturbance force. Similarly as in the case of disturbance caused by elastic force the strategy includes the initial stage when the valve is closed and generated force increases at maximal rate and the stage when valve control provides approximately constant level of generated force (Fig. 8a, b). The first stage is characterized by substantially different shape of forcedisplacement curve and duration due to large influence of the viscous force at the beginning of the process. Consequently, the second stage of the process is characterized by lower level of total force generated by the absorber. Moreover, in contrast to the case of elastic force disturbance, during the second stage of the process at the first part of each control step the valve is repeatedly fully closed in order to compensate the decrease of viscous disturbance force. The sudden increase of the control effort, which appears at certain part of the process is revealed by commutative full closing and opening of the valve at the beginning of the subsequent control steps. Analogously as previously, this phenomenon is caused by too long period when the valve remains closed, too large increase of the pneumatic force at the beginning of the control 


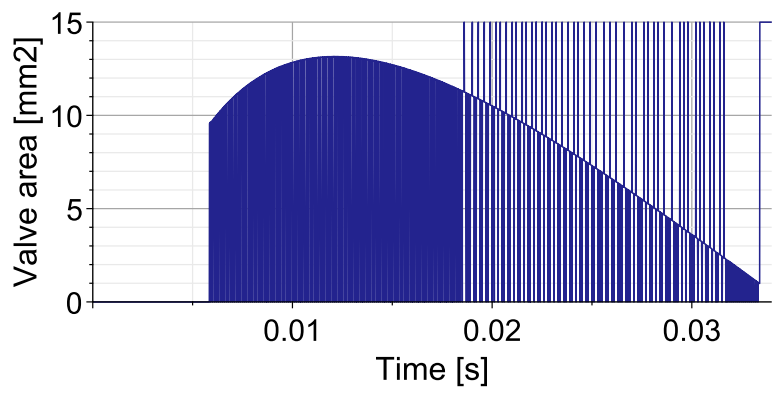

(a)

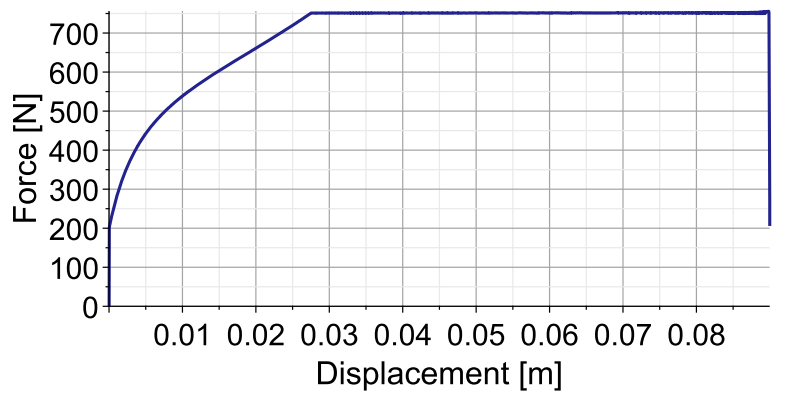

(b)

Fig. 8 Optimal two-stage control strategy, disturbance introduced by viscous force: a change of valve opening area, b change of total force generated by the absorber

step and insufficient compensation of this process by decrease of the viscous force.

In general, the operation of the optimal two-stage control strategy is satisfactory in both tested cases: disturbance by elastic and disturbance by viscous force. It allows to obtain the desired change of total reaction force with a long stage of constant value, providing absorption of the entire impact energy, utilization of the entire absorber's stroke and corresponding minimal level of impacting object's deceleration. In turn, the disadvantage of the system is requirement of conducting intensive control actions with commutative opening and closing of the valve, which increases the required control effort.

\subsection{Control strategy with constant valve opening}

The control strategy with constant valve opening at each control step was also tested using impact excitation and disturbances caused by elastic and viscous forces. The plots of the applied valve opening (Fig. 9a,c) and the corresponding change of total force generated by the absorber (Fig. 9b, d) allow to observe the first stage of the process when valve remains closed in order to enable possibly fast increase of total force up to required value. Once the controller detects that the required level of piston deceleration can be reached at the current control step, the constant value of valve opening is optimized in order to minimize the discrepancy between predicted and optimal value of deceleration at a single control step. Such strategy is continued until the end of the process, which enables maintaining approximately constant level of deceleration until the end of the stroke and absorption of the entire impact energy.

The valve opening and the corresponding impacting object deceleration obtained during ten initial control steps of the second stage of the process are presented in Fig. 10a-d, respectively. The initial oscillations of valve opening occurring both in the case of elastic disturbance (Fig. 10a) and viscous disturbance force (Fig. 10b) are the consequence of applied optimization procedure aimed at minimization of tracking error at the subsequent control steps. Nevertheless, in both cases the applied optimization procedure rapidly stabilizes itself and gradual modifications of valve opening at the subsequent control steps are caused mainly by smooth changes of piston's kinematics. As a result, the valve opening changes regularly during the remaining part of the impact absorption process.

The plots in Fig. 10c and d present the optimal level of impacting object's deceleration calculated using KOC (black), deceleration obtained from the optimization procedure utilizing the predictive model (red) and deceleration obtained from the real model of the system (navy). Let us remind that optimization of valve opening at each control step (Eq. 55) is conducted using the predictive model, which assumes constant value of disturbance during each control step. As a result, the total deviation of the deceleration from the optimal value (the integral in kinematic formulation given by Eq. 55) is minimized only for the predictive model of the system (red and black lines in Fig. 10c and d). When the models of real system with changing disturbance force are applied, the obtained changes of impacting objects' decelerations become 


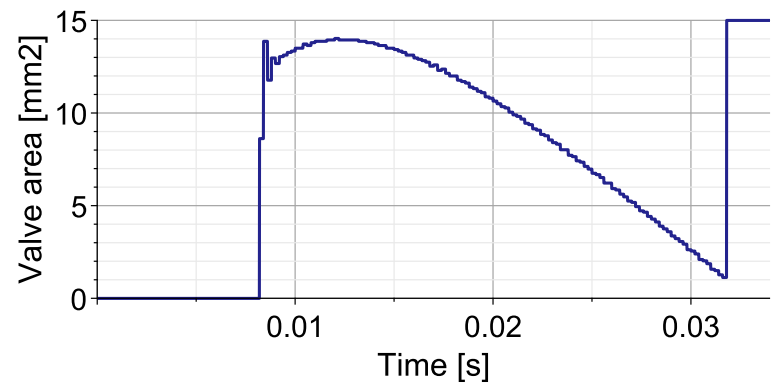

(a)

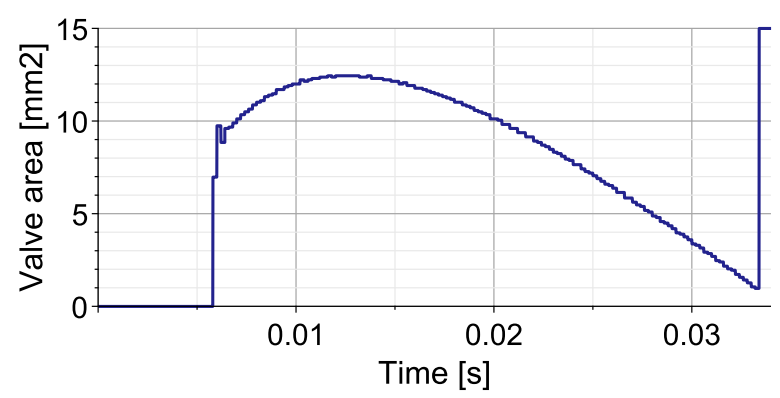

(c)

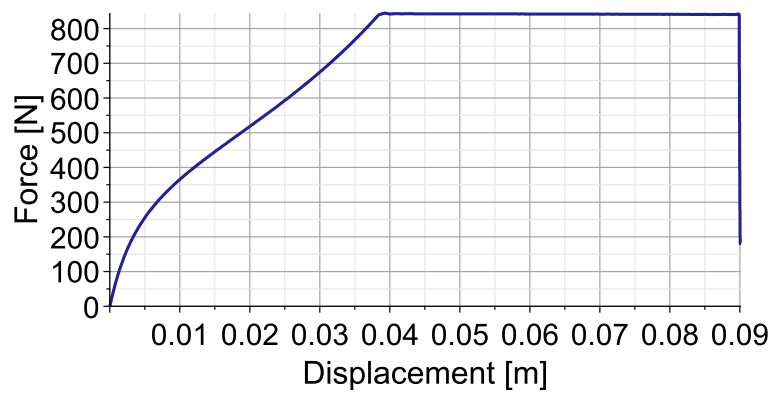

(b)

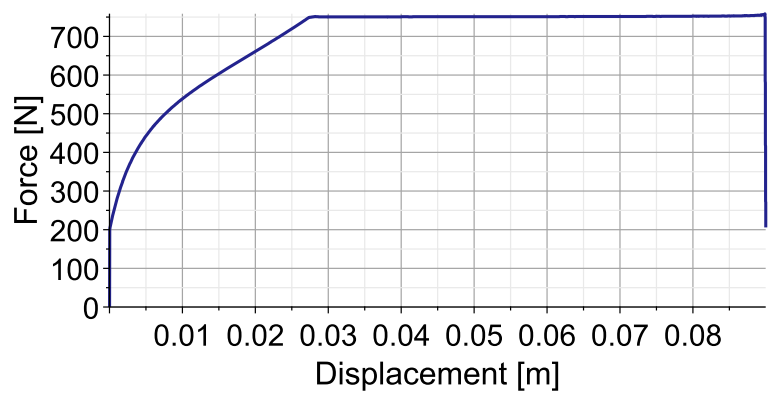

(d)

Fig. 9 Control strategy with constant valve opening at each control step: $\mathbf{a}, \mathbf{b}$ the case of disturbance caused by elastic force; $\mathbf{c}, \mathbf{d}$ the case of disturbance caused by viscous force

slightly different (navy and black lines in Fig. 10c and d). In the case of elastic disturbance the additional elastic force is larger than in the predictive model and real obtained deceleration is larger than the predicted one. In turn, in the case of viscous disturbance the additional viscous force is smaller than in the predictive model and real obtained deceleration is smaller than the predicted one. In other words, the application of the predictive model causes that valve opening determined at each control step is slightly too small in the case of elastic disturbance and slightly too large in the case of viscous disturbance. Nevertheless, the applied strategy causes that during the entire second stage of the process the obtained deceleration remains close to the theoretical optimal value.

\subsection{Control strategy with assumed kinematics}

The similar analysis was conducted for single-stage control strategy with assumed kinematics at each control step. The simulations were performed both for the case of unknown elastic disturbance force (Fig. 11a, b) and viscous disturbance forces (Fig. 11c, d).
The obtained results closely resemble the results achieved previously using the control strategy with constant valve opening at each control step (Sect. 7.2). In particular, the first stage with closed valve provides fast increase of generated force, while the second stage ensures approximately constant level of generated force. The substantial difference between control strategy with constant valve opening and control strategy with assumed kinematics results from the selection of the optimized parameter. In considered approach the valve opening changes during a single control step in order to provide preliminary assumed optimal change of impacting object's deceleration. As a result, the smooth change of the valve opening without jumps between the subsequent steps is observed during the main part of the impact absorption process (Fig. 11a, c). The applied change of valve opening instantly compensates disturbance forces occurring in the system and, in contrast to optimal two-stage method, no additional actions based on sudden opening or closing of the valve are required.

The control strategy with assumed kinematics possesses many features of the control strategy with constant valve opening. In particular, the initial oscillations of valve opening at the beginning of the 


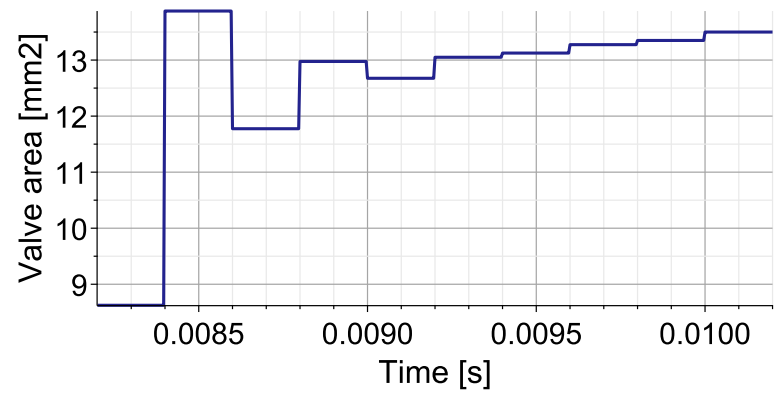

(a)

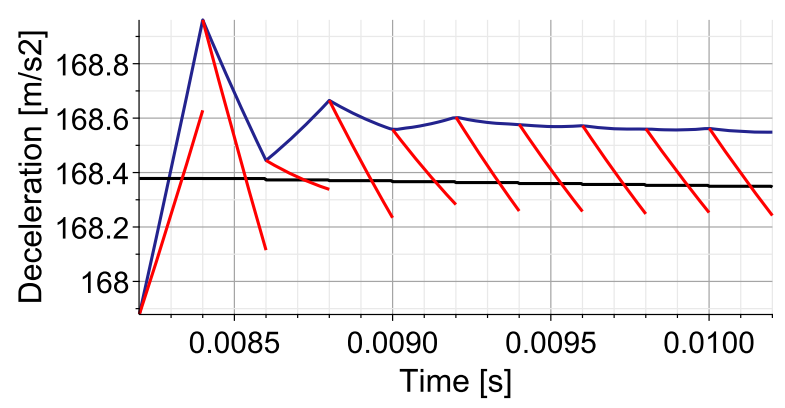

(c)

Fig. 10 Control strategy with constant valve opening: a, b valve opening in the case of elastic and viscous disturbance; c, d obtained deceleration (navy), optimal deceleration

second stage of the process can be observed for both types of considered disturbances (Fig. 12a, b), but they disappear after several milliseconds of the process. The plots in Fig. 12c and d present the optimal level of impacting object's deceleration calculated using KOC (black), the assumed linear change of deceleration at each control step (red) and deceleration obtained from the real model of the system (navy). It can be noticed that all these plots are very similar as in the case of control strategy with constant valve opening (cf. Figures $12 \mathrm{c}, \mathrm{d}$ and 10c, d). Since the optimization of deceleration at each control step (Eq. 59) is conducted using predictive model with constant value of disturbance force, the deviation from the optimal deceleration is minimized for the predictive model (red and black lines in Fig. 12c and d), but not for the real system (navy and black lines in Fig. $12 \mathrm{c}$ and d). The changes of disturbance forces in real system cause that the valve opening is too small in the case of elastic disturbance force and too large in the case of viscous disturbance force. Consequently, the obtained decelerations are larger or smaller than the predicted ones. Nevertheless, the obtained

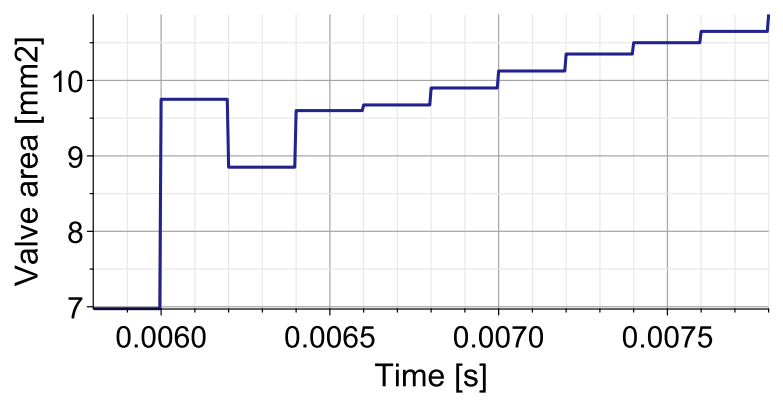

(b)

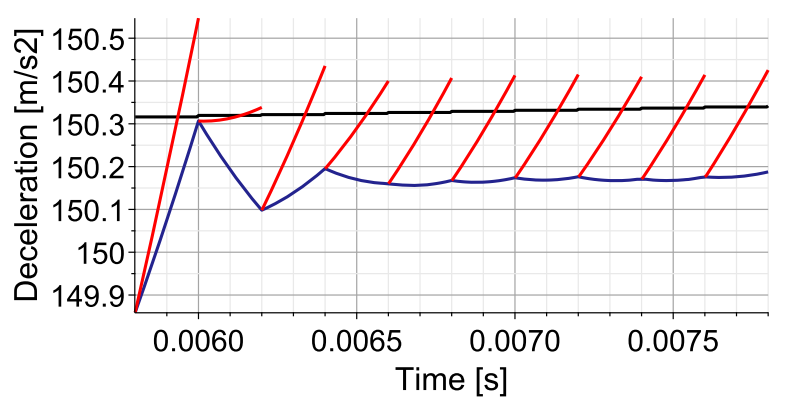

(d)

determined from KOC (black), deceleration obtained from predictive model (red) in the case of elastic and viscous disturbance

decelerations always remain close to the theoretical optimal value.

Both in the system with elastic disturbance and the system with viscous disturbance the actual value of impacting object's deceleration converges to the optimal deceleration value determined from the KOC (Fig. 13a, b). The additional effect is that in the case of elastic disturbance the optimal deceleration permanently decreases since real value of deceleration is always too large, while in the case of viscous disturbance the optimal deceleration permanently increases since real value of deceleration during the entire process is always too small. However, such effect is expected to disappear in the case of random disturbance with zero expected value.

\subsection{Comparison of single-stage control methods}

The detailed comparison of the initial part of the timehistory of valve opening and resulting force obtained from the control strategy with constant valve opening and the strategy with assumed kinematics is presented in Fig. 14a, b for the case of elastic disturbance and in 


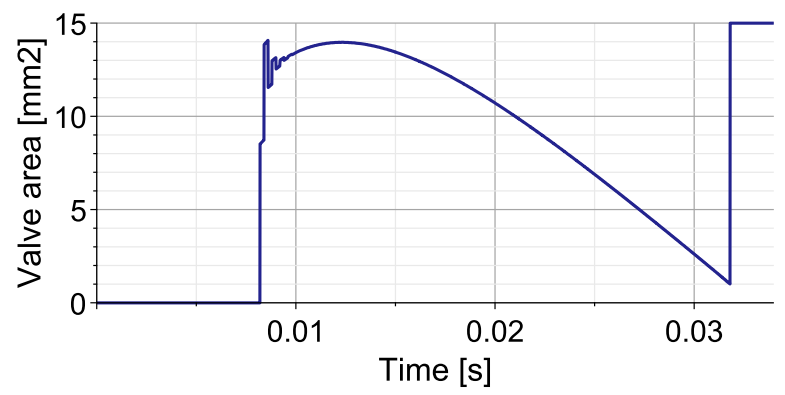

(a)

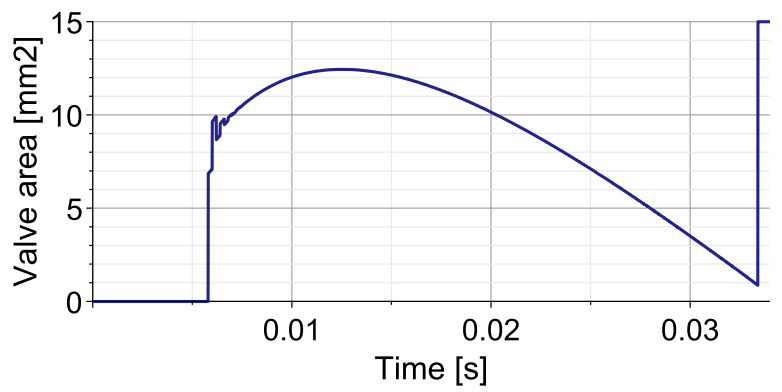

(c)

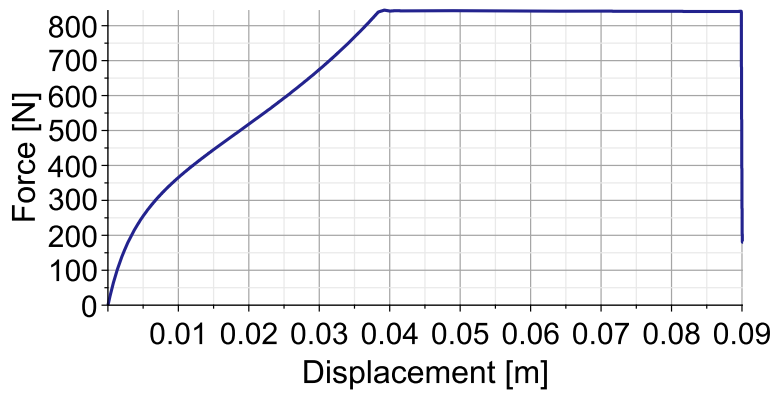

(b)

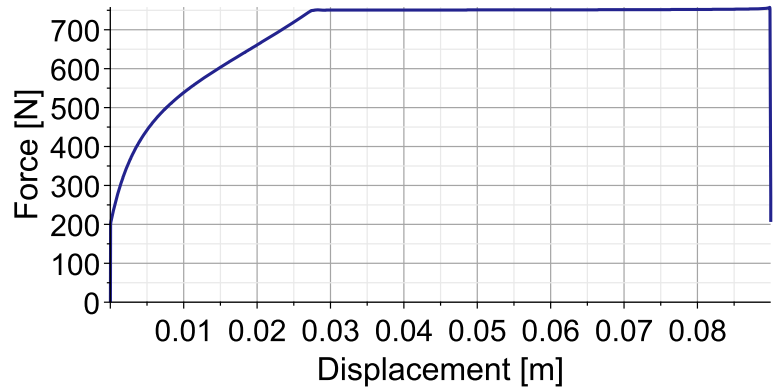

(d)

Fig. 11 Control strategy with assumed kinematics at each control step: a, b the case of disturbance caused by elastic force; $\mathbf{c}, \mathbf{d}$ the case of disturbance caused by viscous force

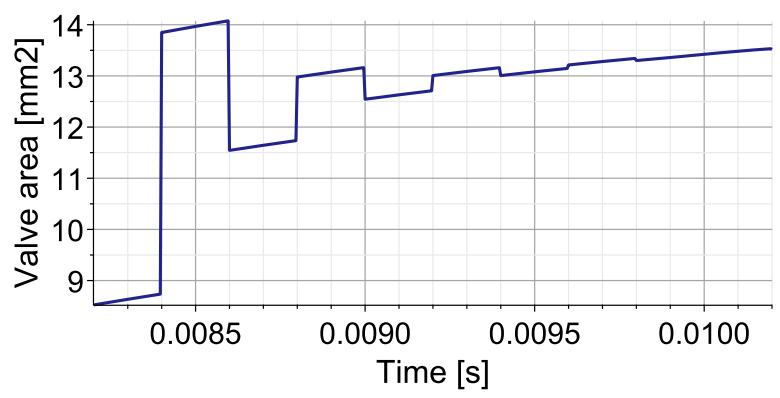

(a)

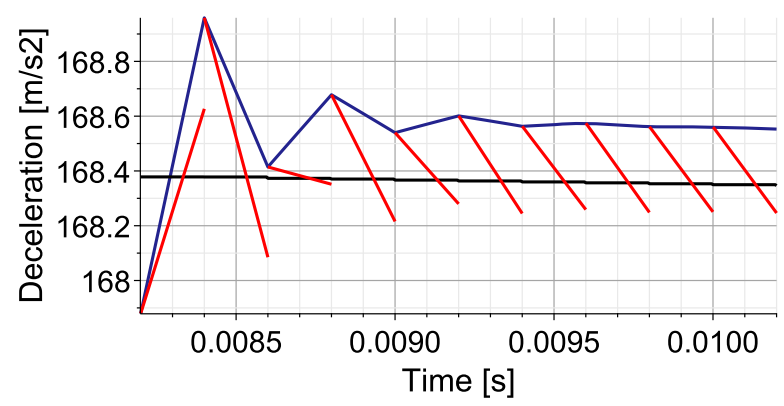

(c)

Fig. 12 Control strategy with assumed kinematics at each control step: a, b valve opening in the case of elastic and viscous disturbance force; c, d obtained deceleration (navy), optimal

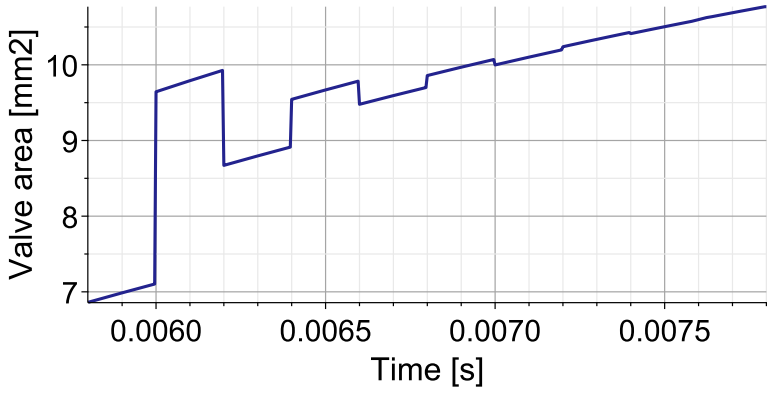

(b)

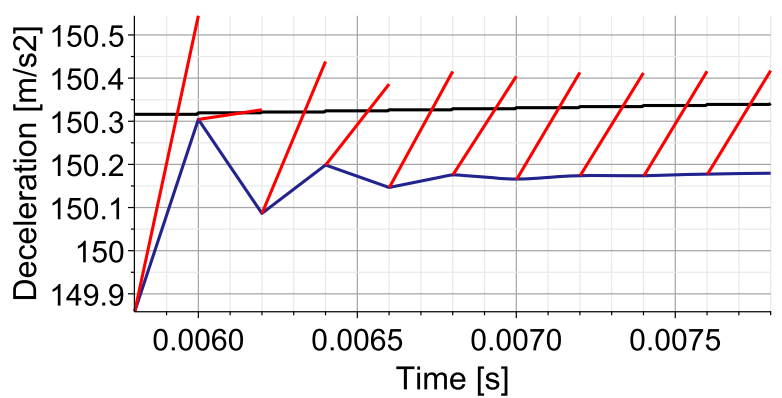

(d)

deceleration determined from KOC (black) and deceleration obtained from predictive model (red) in the case of elastic and viscous disturbance force 


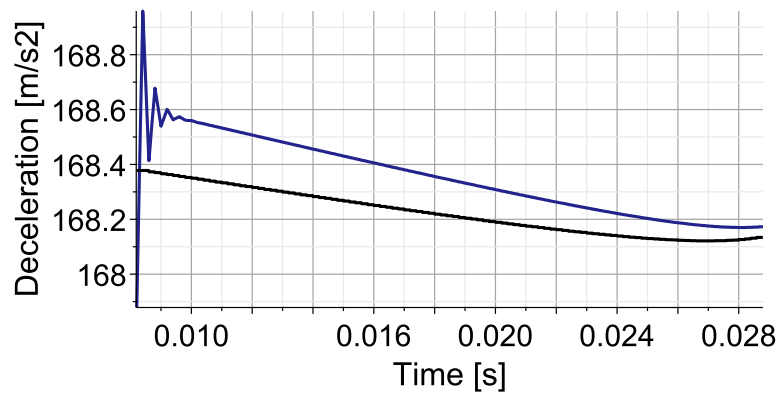

(a)

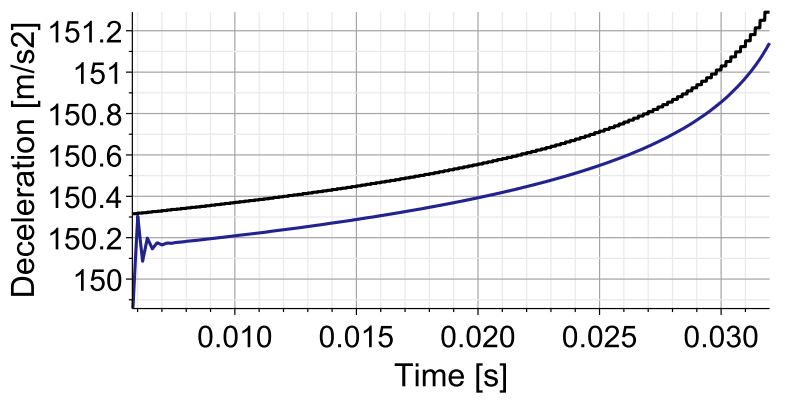

(b)

Fig. 13 Control strategy with assumed kinematics at each control step: convergence of the actual deceleration value (navy) to the optimal deceleration value (black) determined using KOC, obtained in the case of elastic (a) and viscous disturbance force (b)

Fig. 14c, $\mathrm{d}$ for the case of viscous disturbance. The plots show the initial ten control steps of the second stage of impact absorption process. It can be clearly seen that the strategy with assumed kinematics results in very similar opening of the valve as the strategy with constant valve opening. However, the continuous change of valve opening during a single control step causes that the valve opening rapidly becomes continuous and smooth between the steps. Also the change of impacting object's deceleration obtained

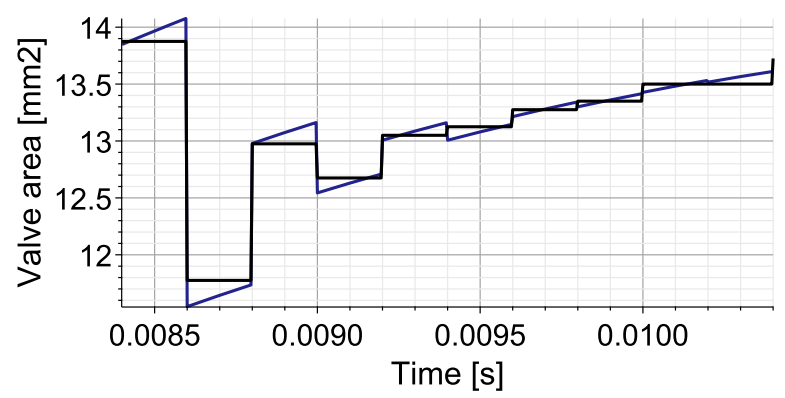

(a)

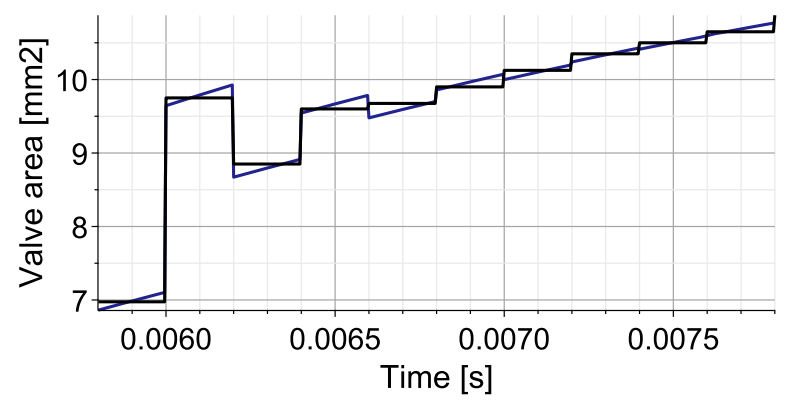

(c) using both control methods is very similar and arising discrepancies are smaller than change of the optimal deceleration during the process.

In general, the operation of the control strategy with constant valve opening and the control strategy with assumed kinematics is satisfactory both in terms of obtained total force generated by the absorber and the corresponding control actions. Both proposed strategies allow to obtain desired change of total generated force, which ensures utilization of the entire stroke and

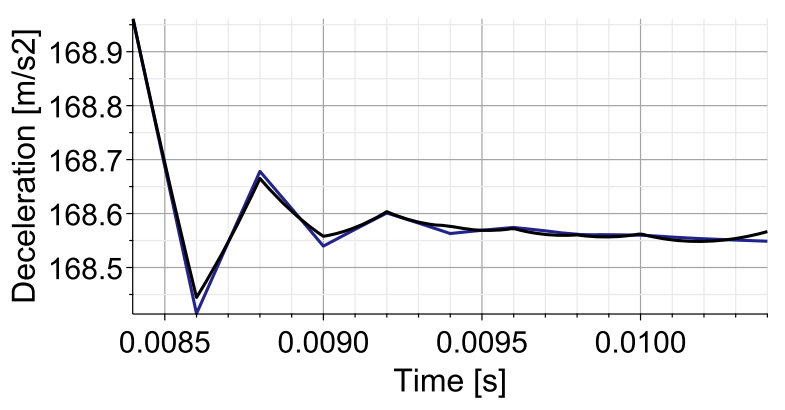

(b)

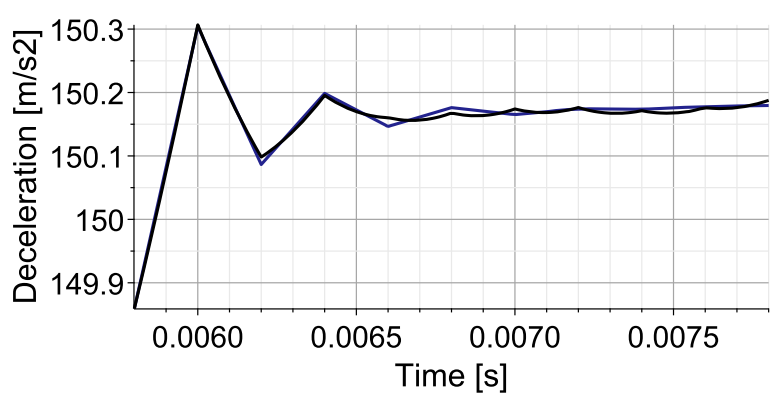

(d)

Fig. 14 Comparison of the initial part of the second stage of control strategy with constant valve opening (black) and control strategy with assumed kinematics (navy): $\mathbf{a}, \mathbf{b}$ elastic disturbance force, $\mathbf{c}, \mathbf{d}$ viscous disturbance force 
minimal value of obtained deceleration. They operate properly under disturbance caused by elastic force, disturbance caused by viscous force and thus they are expected to operate properly in the case of arbitrary type of disturbance forces.

The strategy with constant valve opening does not require change of valve opening during a single control step, but requires small step changes of constant valve openings between the control steps during the entire process. In turn, the strategy with assumed kinematics requires very precise changes of valve opening at each control step, but during the main part of the process the valve opening changes smoothly and remains continuous between the control steps. The disadvantage of the strategy with constant valve opening is the requirement of determining control using optimization procedure. This drawback is eliminated in the control strategy with assumed kinematics where the control is determined analytically in terms of actual system state and optimal kinematics.

\section{Conclusion}

Presented theoretical considerations and results of numerical simulations prove the possibility of successful application of the innovative Identification-based Predictive Control (IPC) methods for efficient solution of the adaptive impact mitigation problem. The proposed methods effectively combine the concept of on-line identification with the concept of Model Predictive Control. Real-time identification of external forces and system disturbances allows to develop the efficient predictive model used for forecasting the system response at the subsequent control steps. In turn, repeated solving of the control problem formulated at each control step for the finite time horizon enables reduction of the computational cost and compensation of the system disturbances. Moreover, application of the kinematic optimality condition, which is recalculated at each control step and incorporated into the successively solved control problems, allows for efficient tracking of the globally optimal solution.

Three different implementations of the IPC presented in this paper directly follow the above paradigm of adaptive predictive control. The optimal two-stage method provides the best possible solution of the control problem, but requires intensive control actions during the entire impact absorption process. The method with constant valve opening provides very similar response, but requires smaller control modifications with gradual change of valve opening. The method with assumed kinematics provides large versatility due to possibility of adopting various functions describing the system kinematics and it results in relatively smooth change of valve opening. Moreover, the suboptimal control can be determined analytically at a low numerical cost.

The proposed innovative control methods provide fully adaptive operation of semi-active shock-absorbers and efficient mitigation of dynamic excitations under the presence of disturbances. The exceptional capabilities of the described control strategies cause that they can be widely applied in various semi-active shock-absorbing devices and used in many branches of engineering. Practical implementation of the proposed IPC methods requires the use of very fast actuators, which can be based on piezoelectric or magnetorheological valves with electromagnets, and efficient data processing systems, such as FPGA modules. As both the executive systems and the data processing and control systems have been successfully applied for adaptive impact mitigation problems, it can be concluded that the proposed Identification-based Predictive Control is feasible. Experimental validation of the proposed approach constitutes a goal of future research of the authors.

Acknowledgements The support of the National Science Centre, Poland, granted through the Agreement 2018/31/D/ST8/ 03178, and the National Centre for Research and Development (NCBiR) granted through the Agreement LIDER/13/0063/L-10/ $18 /$ NCBR/2019 is gratefully acknowledged. RF is supported by the Foundation for Polish Science (FNP) under the START scholarship.

\section{Compliance with ethical standards}

Conflict of interest The authors declare that they have no conflict of interest.

Open Access This article is licensed under a Creative Commons Attribution 4.0 International License, which permits use, sharing, adaptation, distribution and reproduction in any medium or format, as long as you give appropriate credit to the original author(s) and the source, provide a link to the Creative Commons licence, and indicate if changes were made. The images or other third party material in this article are included in the article's Creative Commons licence, unless indicated otherwise in a credit line to the material. If material is not included in the article's Creative Commons licence and your intended use is not permitted by statutory regulation or exceeds 
the permitted use, you will need to obtain permission directly from the copyright holder. To view a copy of this licence, visit http://creativecommons.org/licenses/by/4.0/.

\section{References}

1. Ghiringhelli GL (2000) Testing of semi-active landing gear control for a general aviation aircraft. AIAA J Aircraft 37(4):606-616

2. Mikułowski G, Holnicki-Szulc J (2007) Adaptive landing gear concept - feedback control validation. Smart Mater Struct 16:2146-2158

3. Wang H, Rui Q, Hong H, Li J (2017) Airdrop recovery systems with self-inflating airbag: modeling and analysis, 1st edn. National Defense Industry Press, Beijing

4. Baruh H, Elsayed EA (2018) Experimental design of a folded-structure energy-absorption system. Int J Mater Prod Technol 56(4):341-362

5. Wołejsza Z, Holnicki-Szulc J, Graczykowski C, Hinc K, Faraj R, Kowalski T, Mikułowski G, Kaźmierczak K, Wiszowaty R, Pawłowski P (2018) Dynamics and control of adaptive airbags for UAV impact protection. In: ISMA 2018/USD 2018, international conference on noise and vibration engineering/international conference on uncertainty in structural dynamics, Leuven, Belgium, pp 3661-3670

6. Maeda T, Otsuki M, Hashimoto T (2019) Protection against overturning of a lunar-planetary lander using a controlled landing gear. Proc Inst Mech Eng Part G J Aerosp Eng 233(2):438-456

7. Jawad SAW (1996) Intelligent hydraulic bumper for frontal collision mitigation. ASME Appl Mech Div 218:181-189

8. Woo D, Choi S-B, Choi YT, Wereley NM (2007) Frontal crash mitigation using MR impact damper for controllable bumper. J Intell Mater Syst Struct 18(12):1211-1215

9. Myung-Hyun N, Sang-Youl L (2018) Parametric impact performances in a new type crash cushion barrier system using an energy absorption pipe. Int J Crashworth, pp 1-14, in press

10. Richert J, Coutellier D, Götz C, Eberle W (2007) Advanced smart airbags: the solution for real-life safety? Int J Crashworthiness 12:159-171

11. Graczykowski C, Holnicki-Szulc J (2009) Protecting offshore wind turbines against ship impacts by means of adaptive inflatable structures. Shock Vib 16(4): 335-353

12. Spaggiari A, Dragoni E (2012) Efficient dynamic modelling and characterization of a magnetorheological damper. Meccanica 47(8):2041-2054

13. Powers BE, Wereley NM, Choi YT (2016) Analysis of impact loads in a magnetorheological energy absorber using a Bingham plastic model with refined minor loss factors accounting for turbulent transition. Meccanica 51(12):3043-3054

14. Makowski M, Knap L (2014) Reduction of wheel force variations with magnetorheological devices. J Vib Control 20(10)
15. Makowski M, Knap L (2018) Investigation of an off-road vehicle equipped with magnetorheological dampers. Adv Mech Eng 10(5)

16. Lou Z, Ervin R, Filisko F, Winkler C (1993) An electrorheologically controlled semi-active landing gear. SAE Trans 102:334-342

17. Nguyen QH, Choi SB, Park YG (2012) An analytical approach to optimally design of electrorheological fluid damper for vehicle suspension system. Meccanica 47(7):1633-1647

18. Zalewski R, Chodkiewicz P (2016) Semi-active linear vacuum packed particles damper. J Theoret Appl Mech 54(1):311-316. https://doi.org/10.15632/jtam-pl.54.1.311

19. Bartkowski P, Zalewski R (2018) A concept of smart multiaxial impact damper made of vacuum packed particles. In: MATEC web of conferences, 157, Art. No. 05001, https:// doi.org/10.1051/matecconf/201815705001

20. Mikułowski G, Wiszowaty R (2016) Pneumatic adaptive absorber: mathematical modelling with experimental verification. Math Probl Eng 7074206-1-14

21. Wasilewski M, Pisarski D, Bajer CI (2019) Adaptive optimal control for seismically excited structures. Autom Constr 106:1-18

22. Popławski B, Mikułowski G, Mróz A, Jankowski Ł (2018) Decentralized semi-active damping of free structural vibrations by means of structural nodes with an on/off ability to transmit moments. Mech Syst Signal Process 100:926-939

23. Wasilewski M, Pisarski D, Konowrocki R, Bajer CI (2019) A new efficient adaptive control of torsional vibrations induced by switched nonlinear disturbances. Int $\mathbf{J}$ Appl Math Comput Sci 29(2):285-303

24. Holnicki-Szulc J, Graczykowski C, Mikułowski G, Mróz A, Pawłowski P, Wiszowaty R (2015) Adaptive impact absorption - the concept and potential applications. Int J Prot Struct 6(2):357-377

25. Holnicki-Szulc J, Maćkiewicz A, Kołakowski P (1998) Design of adaptive structures for improved load capacity. AIAA J 36(3):471-476

26. Jankowski Ł, Graczykowski C, Pawłowski P, Mikułowski G, Ostrowski M, Popławski B, Faraj R, Suwała G, HolnickiSzulc J (2017) Adaptive self-protection against shock and vibration. Adv Sci Technol 101:133-142

27. Sekuła K, Graczykowski C, Holnicki-Szulc J (2013) Online impact load identification. Shock Vib 20(1):123-141

28. Ilchmann A, Sawodny O, Trenn S (2016) Pneumatic cylinders: modelling and feedback force control. Int $\mathrm{J}$ Control 79(6):650-661

29. Wang J, Pu J, Moore P (1999) A practical control strategy for servo-pneumatic actuator systems. Control Eng Pract 7:1483-1488

30. Faraj R, Graczykowski C, Holnicki-Szulc J (2018) Adaptable pneumatic shock absorber. J Vib Control 25(3):711-721

31. Ghiringhelli GL, Gualdi S (2004) Evaluation of a landing gear semi-active control system for complete aircraft landing. Aerotecnica Missili e Spazio 83:21-31

32. Wang H, Xing JT, Price WG, Li W (2008) An investigation of an active landing gear system to reduce aircraft vibrations caused by landing impacts and runway excitations. J Sound Vib 317:50-66 
33. Sivakumar S, Haran A (2013) Mathematical model and vibration analysis of aircraft with active landing gears. J Vib Control 21(2):229-245

34. Hui L, Hongbin G, Dawei C (2008) Application of highspeed solenoid valve to the semi-active control of landing gear. Chin J Aeronaut 21(3):232-240

35. Graczykowski C, Faraj R (2019) Development of control systems for fluid-based adaptive impact absorbers. Mech Syst Signal Process 122(2019):622-641

36. Faraj R, Graczykowski C (2019) Hybrid prediction control for self-adaptive fluid-based shock-absorbers. J Sound Vib 449:427-446
37. Faraj R, Mikułowski G, Wiszowaty R (2020) Smart mater Struct 29(11):115008. https://doi.org/10.1088/1361-665X/ ab9adc

38. Graczykowski C, Pawłowski P (2017) Exact physical model of magnetorheological damper. Appl Math Model. https:// doi.org/10.1016/j.apm.2017.02.035

39. Faraj R (2019) Development of self-adaptive systems for mitigation of response under dynamic excitation, $\mathrm{PhD}$ Thesis, IPPT PAN

Publisher's Note Springer Nature remains neutral with regard to jurisdictional claims in published maps and institutional affiliations. 